\title{
All-atom structural models of insulin binding to the insulin receptor in the presence of a tandem hormone-binding element
}

Harish Vashisth*

Department of Chemistry and Biophysics Program, University of Michigan, Ann Arbor, MI

Cameron F. Abrams ${ }^{\dagger}$

Department of Chemical and Biological Engineering, Drexel University, Philadelphia, PA, USA

*E-mail address: harishv@umich.edu

†E-mail address: cfa22@drexel.edu 


\section{SUPPLEMENTAL METHODS}

Monte Carlo (MC) docking. Initial coordinates for the receptor were taken from our previous insulin/IR $\Delta \beta$ structural models, ${ }^{1}$ which were prepared based upon a 3.8- $\AA$-resolution $X$-ray crystal structure of the human insulin receptor ectodomain (PDB code 2DTG). ${ }^{2}$ Two different sets of IR $\Delta \beta$ coordinates, corresponding to each $\mathrm{T}$ and $\mathrm{R}$ insulin-docked model, were extracted. The CT-peptide was then incorporated in these two IR $\Delta \beta$ conformations by extracting the initial coordinates of CT from the refined IR $\Delta \beta$ structure of Smith et al. ${ }^{3}$ (PDB code 3LOH) by aligning the L1 domain (1-157; $\mathrm{C}_{\alpha}$ atoms) of refined IR $\Delta \beta$ with the L1 domain (1-157; $\mathrm{C}_{\alpha}$ atoms) of each IR $\Delta \beta$ conformation. The configurations for $\mathrm{T}$ and $\mathrm{R}$ insulin were sampled from an ensemble of solution conformations of each previously generated via two separate $\sim 1$ ns MD equilibration trajectories. ${ }^{1}$

We implemented the docking algorithm using a Tcl script in $\mathrm{VMD}^{4}$ and the source code is now available. ${ }^{5}$ Our original MC algorithm ${ }^{1,6}$ allows exhaustive conformational sampling from solution MD equilibration trajectories of both ligand and the receptor, but such conformation sampling is only allowed for insulin in this work because the receptor conformations that allow binding of insulin are already known from our previous work. ${ }^{1}$ The docking algorithm minimizes the van der Waals and electrostatic interaction energies between all ligand atoms and atoms we defined as forming a binding pocket. The docking was performed in the previously observed accessible binding pocket formed by the (L1-CR-L2) and (F1-F2-F3)' domains (prime denotes domains from the subunit which does not contribute the L1 domain to insulin accessible pocket). ${ }^{1}$ Specifically, we included the following IR $\Delta \beta$ residues in the MC energy calculation: (i) the L1 residues 12-16, 32, 34, 36-39, 59, 60, 62, 64, 65, 67, 86-91, 94, 96, 97, 118, 120, 121, 144, 146, 147, (ii) the CR residues 270, 271-275, 286-288, (iii) the L2 residues 315-320, (iv) the F1 residues 481-484, 554-557, 593-597, (v) the F2 residues 619-622, and 796. The CT-peptide residues 693, 696, 697, 699, 700, 703, 707, 708, and 710 were included in each docking. The remaining CT residues 694, 695, 698, 701, 702, 705, 706, and 709 are not solvent exposed because they are sandwiched between the CT-peptide and the L1 surface, and therefore were not included in any docking. The trial moves in Metropolis MC sampling consisted of (i) global orientation angle displacements of up to $2^{\circ}$ (about the COM of each ligand), (ii) translational displacements of each ligand up to $1 \AA$, and (iii) conformational sampling from MD equilibration trajectories of each insulin. The initial positions of ligands were randomly generated by aligning the center of mass of each ligand with the geometric center of the binding pocket region. We performed a total of 10000 to $20000 \mathrm{MC}$ cycles per docking trial, which resulted in 10 docked configurations for each insulin in the accessible pocket, and no successful dockings in the inaccessible pocket. We also note that docking trials into unequilibrated refined IR $\Delta \beta$ (PDB code $3 \mathrm{LOH}$ ) were also unsuccessful.

MC-docking of R-insulin in the displaced-CT model was performed as follows. The positions of R-insulin and the CT peptide in the lowest-free energy $\mathrm{R} / \mathrm{IR} \Delta \beta$ configuration (img18; Fig. S5) were first swapped based upon the $\mathrm{C}_{\alpha}$-alignment of the CT peptide residues 693-710 and insulin residues B1-B18. The conformational sampling of ligand was carried out from a 10-ns long MD trajectory of R-insulin that showed separation of the C-terminal tail residues (B22-B30; Fig. S15). The conformational sampling of receptor was carried out from a previously generated $\sim 70$-ns long MD equilibration of IR $\Delta \beta .^{1}$ Same residues of receptor and ligand as described above were included in the energy calculation during MC docking protocol. We generated 10 docked configurations of R-insulin/CT in the open pocket of IR $\Delta \beta$ that are shown in Fig. $5 B$ (see main manuscript). Details on the thermodynamic characterization of these 10 docked models via string method are presented below.

Post-docking molecular dynamics (MD) simulations. Out of all docked models, we chose those two final models (one for each $\mathrm{T}$ and $\mathrm{R}$ insulin) for structural equilibration that displayed highest degree of agreement with experimentally known interactions between insulin and IR. The final $\mathrm{T}$ and $\mathrm{R}$ insulin-docked models have ligand/receptor interaction energies of $-64 \mathrm{kcal} / \mathrm{mol}$ and $-42 \mathrm{kcal} / \mathrm{mol}$, respectively. Each of these two models was further equilibrated via MD simulations. Each insulin/IR $\Delta \beta$ complex was solvated via explicit TIP3P water in a periodic box of dimensions $160 \AA \times 165 \AA \times 125 \AA$. All hydrogen atoms were included, and each system was energy minimized via 2000 cycles of conjugate-gradient optimization. The systems were neutralized with random placement of $\mathrm{Na}^{+}$and $\mathrm{Cl}^{-}$ions. The final $\mathrm{T}$ and $\mathrm{R}$ insulin-docked systems have 331,048 and 303,281 atoms, 
respectively. The temperature was maintained at 300K using the Langevin thermostat with damping coefficient of 5 $\mathrm{ps}^{-1}$. Non-bonded interactions were cut-off beyond $10 \AA$ with smooth switching taking effect at $8.0 \AA$. Long-range electrostatic interactions were handled using the particle mesh Ewald (PME) method with a $1 \AA$ grid spacing. For each system, the equilibration phase was carried out for 11-ns in the NVT ensemble using a 2-fs time step with active rigid bonds. We generated all MD trajectories using NAMDv2. $8^{7}$ using the CHARMM force field ${ }^{8}$ with the CMAP correction. ${ }^{9}$ The displaced-CT model of R-insulin/IR $\Delta \beta$ (see Fig. 5 of the main manuscript) has 307,583 atoms in total, and was equilibrated via a 12-ns unbiased MD simulation following similar protocols as described above.

Temperature-accelerated molecular dynamics (TAMD). We have used TAMD simulations for the conformational sampling of the C-terminus of the B-chain of each insulin molecule. TAMD was originally proposed by Maragliano and Vanden-Eijnden, ${ }^{10,11}$ and has been reviewed in detail previously. ${ }^{12}$ In this work, we have used TAMD as it was originally implemented in NAMD by Abrams and Vanden-Eijnden ${ }^{13}$ as a unique method to study the large-scale conformational changes in proteins. Therefore, we briefly describe the underlying equations here. The coupled system of equations describing TAMD are as follows:

$$
\begin{aligned}
m_{i} \ddot{x}_{i} & =-\frac{\partial V(x)}{\partial x_{i}}-\kappa \sum_{j=1}^{m}\left[\theta_{j}^{*}(x)-\theta_{j}\right] \frac{\partial \theta_{j}^{*}(x)}{\partial x_{i}}-\gamma m_{i} \dot{x}_{i}+\eta_{i}(t ; \beta) \\
\bar{\gamma} \bar{m}_{j} \dot{\theta}_{j} & =\kappa\left[\theta_{j}^{*}(x)-\theta_{j}\right]+\xi_{j}(t ; \bar{\beta})
\end{aligned}
$$

where $\theta^{*}(x)=\left(\theta_{1}^{*}(x), \theta_{2}^{*}(x), \ldots \ldots, \theta_{m}^{*}(x)\right)$ are collective variables that are functions of atom Cartesian coordinates, $m_{i}$ and $\bar{m}_{j}$ are the masses of $x_{i}$ and $\theta_{j}, V(x)$ is the interatomic MD potential, $\kappa$ is the "coupling spring-constant", $\gamma$ is the Langevin friction coefficient, $\eta$ is the white noise satisfying fluctuation-dissipation theorem at physical temperature $\beta^{-1}, \bar{\gamma}$ and $\xi$ respectively are fictitious friction and thermal noise at artificial temperature $\bar{\beta}^{-1}$.

The aforementioned set of equations describe the motion of $x(t)$ and $\theta(t)$ over the extended potential

$$
U_{\kappa}(x, \theta)=V(x)+\frac{\kappa}{2} \sum_{j=1}^{m}\left[\theta_{j}^{*}(x)-\theta_{j}\right]^{2}
$$

As shown previously, ${ }^{10}$ by choosing $\kappa$ so that $\theta^{*}(x(t)) \approx \theta(t)$ and fictitious friction coefficient $\bar{\gamma}$ so that $\theta$ moves slower than $x$, we can generate a trajectory at artificial temperature $\bar{\beta}^{-1}$ subject to the free energy computed at the physical temperature $\beta^{-1}$. Hence, we have chosen a TAMD friction $\bar{\gamma}$ of $500 \mathrm{ps}^{-1}$ and a spring constant $\kappa$ of $100 \mathrm{kcal} / \mathrm{mol} \cdot \AA^{2}$. Here, we choose the Cartesian coordinates of centers of mass of spatially contiguous groups of residues as CVs. Particularly, the residues in the C-terminus of B-chain (23-30) were divided into four subgroups (4 groups of 2 residues each) and hence $12 \mathrm{CVs}$. Therefore, the conformational sampling of only B-chain C-terminus was accelerated via TAMD at a fictitious thermal energy $\left(\bar{\beta}^{-1}\right)$ of $6 \mathrm{kcal} / \mathrm{mol}$ and the remaining atoms in the system evolved under the CHARMM force field. We have also carried out two additional TAMD runs with different initial velocities, and at a fictitious thermal energy of $6 \mathrm{kcal} / \mathrm{mol}$ and $5 \mathrm{kcal} / \mathrm{mol}$. All other parameters were identical to the original TAMD run. We describe data from these TAMD trajectories in the Supplemental Results.

String method calculations for conformational change in the C-terminus of the B-chain of insulin. We have recently used TAMD in combination with the string method to characterize a large-scale conformational change in the insulin receptor kinase domain (IRKD), and hence, implementation details remain similar to as described before. ${ }^{14}$ The string method ${ }^{15-20}$ is a technique to compute the minimum free energy path (MFEP) in a large but finite set of CVs, functions of the atomic Cartesian coordinates. A MFEP is defined as the curve whose tangent is always parallel to the gradient of the free energy (the mean force) times a metric factor which, for the case of linear CVs used here, is constant and diagonal. The algorithm works by iteratively refining (vide infra) an initial "string", i.e. a collection of discrete configurations of the system known as images. The string method and its variants have been used previously for many diverse problems. ${ }^{21-23}$ 
Initial string. The images in the initial strings in our work were chosen from the $\sim 40$ ns long TAMD simulations of the C-terminus of the B-chain of each insulin (vide supra). Because TAMD trajectories can spend significant time exploring the phase-space locally, not every configuration in a TAMD trajectory contributes to the reactive trajectory. Hence, we carefully pruned the TAMD trajectory such that configurations that take the system away from the initial state, yet toward the final state are chosen. The configurations chosen this way, although unique and discrete, are not equidistant in CV-space, which is a requirement for the iterative string evolution. Hence, using the re-parameterization procedure described in the original string method work, ${ }^{19}$ we created $25(0,1,2, \cdots, 24)$ equidistant images that form our initial string for each insulin/receptor complex. Because the initial string is merely a rough estimate of MFEP, the iterative refinement of this initial guess toward MFEP may still be required. We have found that TAMD-generated paths are statistically relevant and closely resemble MFEP. ${ }^{14}$ Hence, we suggest that TAMD is an ideally suited method for generating such initial paths, because the conformational transitions in TAMD are obtained from the exploration of the free-energy landscape at the physical temperature $\beta^{-1} \cdot{ }^{11,13,19}$

String evolution. Strings were evolved by computing the mean force at each image along the strings at each iteration. One can obtain the mean force for each image from restrained MD simulations, i.e. using the potential defined in eq. S2 but with $\theta_{j}$ fixed, and using the estimator described in Maragliano et al. ${ }^{19}$ In this work, we used $100 \mathrm{ps}$ long restrained simulations with force constant $k=5 \mathrm{kcal} / \mathrm{mol} \cdot \AA^{2}$, after having equilibrated the system at each image using a significantly higher $k$. The $100 \mathrm{ps}$ long trajectories were used for the mean force estimation at each image. However, the mean forces in the final converged strings were estimated using significantly longer trajectories for each image. Weak harmonic restraints were also used on the receptor backbone and part of the insulin molecules (except B21-B30) to avoid net translation and rotation during restrained runs, because CVs in our case are not "internal" coordinates. We updated the images in each string by measuring the mean restraining force (or the negative gradient of the free energy $F)$ for each $C V\left(\theta_{j}^{*}\right)$ using the following equation:

$$
\vec{f}_{\theta_{j}^{*}}^{m e a n}=-\frac{\partial F\left(\theta^{*}\right)}{\partial \theta_{j}^{*}} \approx k\left(\left\langle\theta_{j}^{*}\right\rangle-\theta_{j}^{*}\right)
$$

We also note that the string images for each insulin were updated using the full force computed at each image and not its orthogonal component, since using the full force simplifies and improves ${ }^{18}$ the original implementation. Hence, it was not necessary to smooth the strings in this work (a smoothing step was used in the projected-force evolution of the string to improve the tangent calculation). All string calculations were carried out with explicitly solvated and ionized images in NAMDv2.8 using the CHARMM force-field (vide supra).

Potential of mean force (free-energy) calculations. The free energy profile at each string iteration can be computed by integrating the mean force along the string ${ }^{19}$ using the following equation:

$$
\mathrm{PMF}=\int_{0}^{\alpha}\left[\sum_{j=1}^{N} \vec{f}_{\theta_{j}^{*}}^{m \text { man }} \times t_{\theta_{j}^{*}}\right] d \alpha
$$

where $\mathrm{N}$ is the total number of $\mathrm{CVs}$ (12 here), $\vec{f}_{\theta_{j}^{*}}^{\text {mean }}$ can be computed using equation S3, and $t_{\theta_{j}^{*}}$ is the tangent to the path for that CV. The tangent calculation was performed using the forward difference formula for the first image, the backward difference formula for the last image, and the central difference formula for all other images. The integral was computed using the trapezoidal rule. PMF along the path is then a sum over the product of mean force at each image multiplied by the tangent to the path at that point. We point out that the free-energy profiles computed by the string method are in the space of $\mathrm{CVs}$, which is nonetheless multi-dimensional for each string (12 dimensional here corresponding to $12 \mathrm{CVs}$ ).

String convergence. To assess the convergence to the MFEP, we monitored three properties during successive string iterations for each insulin/IR complex: (a) the free energy profiles, (b) the root-mean square deviation 
(RMSD) of the string in CV-space, (c) the average string RMSD over different iterations. We present these results in Fig. S2 and Fig. S3, while the snaphots of converged images in MFEP are presented in Fig. S4 and Fig. S5. The error bars for PMF were estimated by measuring the statistical fluctuations of each image in the converged string using independent simulations. The last images in each string were also allowed to evolve.

String method calculations for the displaced-CT model. For the thermodynamic characterization of $10 \mathrm{MC}$ docked structural models of R-insulin/IR $\Delta \beta$ complexes, we used the string method in collective variables (vide supra). We first created an initial string containing 10 images corresponding to $10 \mathrm{MC}$ docked configurations. As CVs for the evolution of string, we chose the center-of-mass Cartesian coordinates of R-insulin and CT together, because MC docking was performed on the joint COM of R-insulin and CT. For estimation of the mean force at each image, we used $100 \mathrm{ps}$ long restrained simulations with force constant $k=50 \mathrm{kcal} / \mathrm{mol} \cdot \AA^{2}$, after having equilibrated the system at each image using a significantly higher $k$. The free-energy profiles at each string iteration (Fig. 6A) were computed using same methodologies as described above.

Definitions of observables: Buried surface area (BSA) and binding pocket radius of gyration $\left(R_{g}\right)$. The buried surface area (BSA) between any two domains A and B is computed by subtracting the solvent-accessible surface area (SASA) of the isolated $\mathrm{AB}$ complex from the sum of SASAs of isolated and separate A and B domains:

$$
\mathrm{BSA}(\mathrm{AB})=\mathrm{SASA}(\mathrm{A})+\mathrm{SASA}(\mathrm{B})-\mathrm{SASA}(\mathrm{AB})
$$

All SASA calculations were done with VMD using a probe radius of $1.4 \AA$. Hereafter, when referring to the interface between domains $\mathrm{A}$ and $\mathrm{B}$, we use the notation " $\mathrm{A} / \mathrm{B}$ ". The residues used to measure size of the binding pockets using the radius of gyration $R_{g}$ have been described before. ${ }^{1}$ For any collection of $N$ atoms each having Cartesian position $\mathbf{r}_{i}$ and mass $m_{i}, R_{g}$ is defined as

$$
R_{g}^{2}=\frac{\sum_{i=1}^{N} m_{i}\left(\mathbf{r}_{i}-\mathbf{r}_{\mathrm{CM}}\right)^{2}}{\sum_{i=1}^{N} m_{i}}
$$

where $\mathbf{r}_{\mathrm{CM}}$ is the center of mass of the collection.

Collective variable conformational metrics. We have previously used ${ }^{1}$ identical conformational metrics as described below to characterize apo-IR $\Delta \beta$. In order to characterize the overall conformational variability of the IR $\Delta \beta$ ectodomain observed during the MD and TAMD simulations, we defined a set of collective variables based on the center-of-mass positions of each domain and the locations of the hinges between them. Each subunit is conceptualized as a linear chain of "mapping points" that correspond to positions of domains and the hinges connecting them. Domains L1, L2, F1, F2, and F3 of each subunit contribute a mapping point. The L1-L2, F1-F2, and F2-F3 intradomain hinge mapping points are defined as the centers of mass, respectively, of residues 304 to 308; 559, 592, and 621; and $638,775,807$, and 840. Finally, a common "apex hinge" mapping point connects L2, L2', F1, and F1' and is defined as the center of mass of the four-domain apex. In this manner, the entire IR $\Delta \beta$ structure (more than 25,000 atoms distributed over 1,612 residues) is coarse-grained onto 17 Cartesian positions for conformational analysis. The set of collective variables considered here based on these mapping points are (1) the F1-F2 and F1'-F2' hinge angles; (2) the L1-L2 and L1'-L2' hinge angles; (3) the L2/F1' and L2' /F1 hinge angles, and (4) the L1-L2↔Fn1'-F2' and L1'L2' $\leftrightarrow$ F1-F2 interhinge distances. Hereafter, when referring to the hinge angle due to domains $A$ and $B$, we use the notation "A-B". [Note the labelling distinction between interfaces ("A/B") and hinge angles ("A-B")].

\section{SUPPLEMENTAL RESULTS AND DISCUSSION}

Characterization of receptor dynamics during MD equilibration of MC-docked models of T/IR $\Delta \beta$ and R/IR $\Delta \beta$. We equilibrated our MC docked models of T- and R-insulin for 11-ns via explicit-solvent MD simulations. We show data characterizing the intradomain relaxation and flexibility of the receptor during these simulations for each 
insulin/receptor complex in Fig. S6. Specifically, we show the RMSD traces for backbone $\mathrm{C}_{\alpha}$ atoms of each domain of ligand-bound IR $\Delta \beta$ during the 11-ns long MD trajectories. These data generally indicate that the overall fold of each domain is preserved during MD relaxation given their modest RMSD values between 1 to $5 \AA$. The CR domain and the fibronectin repeats show relatively larger deviations than the L1 and L2 domains, which drift very little in comparison to other domains. The apparent mobility of fibronectin domains could be both due to the absence of membrane-anchors as well as their lower resolution (in comparison to L1-CR-L2) in the crystal structure of apo-IR $\Delta \beta$ (PDB code 2DTG). These flexibility mechanisms are consistent with our earlier work. ${ }^{1}$

We further describe interdomain flexibility of ligand-bound IR $\Delta \beta$ via a coarse-grained representation using collective geometric variables such as interdomain hinge angles and distances defined in terms of Cartesian positions of "mapping points" (see Supplemental Methods for definitions of mapping points). We show the labeled collective variable traces in Fig. S7 for each insulin/IR complex. In general, we observe that the F1-F2 hinges of IR $\Delta \beta$ for each complex tend to stay more open than what is observed in the crystal structure, while the L1-L2 hinges tend to stay contracted in comparison to the crystal structure values. For each complex, the L2-F1 hinges on the apex of receptor ectodomain maintain their crystallographic values throughout equilibration. The distances between F1-F2 and L1-L2 hinges on both sides for T/IR complex decrease in the beginning before reaching values observed in the crystal structure towards the end, but the same hinges for R/IR do not appreciably change during equilibration.

Consistent with inter-domain hinge-angle fluctuations are buried surface areas (BSA) and the size of each binding pocket, which jointly characterize intersubunit flexibility (Fig. S8A). We observe that BSA values at the L1/F2 interfaces on both sides (for each complex) are higher than their values in the crystal structure. At the L2/F1 interfaces, BSA values for the T/IR complex are higher than their values in the crystal structure, while for R/IR complex, BSA values decrease on both sides only to regain their crystallographic values. Both binding pockets in the R/IR complex tend to become equally open as seen in the crystal structure, however, the size of pockets does not change appreciably for the T/IR complex during MD equilibration.

Characterization of bound ligands during MD equilibration of MC-docked models of T/IR $\Delta \beta$ and R/IR $\Delta \beta$. To characterize the change in ligands during MD equilibration, we present same metrics as presented in Fig. $2 A$ (see main manuscript). Specifically, we do not see any appreciable change in the conformation of bound insulin molecules as indicated by RMSD values of the C-terminal tail (B21-B30) of each insulin (1); Fig. S8B), also resulting in no exposure of BSA between the C-terminal tail and the rest of insulin molecules (2); Fig. S8B). During MD equilibrations, no increased registry with the L1 domain is seen for either insulin as indicated by BSA between each insulin and L1 (3; Fig. S8B). The conformation of CT fluctuates slightly more in the T/IR complex than in the R/IR complex (4) in Fig. S8B, and snapshots of CT in Fig. S8C). Two different views of the conformations of docked insulin molecules after MD equilibration are shown in Fig. S9.

Characterization of receptor dynamics during TAMD simulations of MC-docked and MD-equilibrated models of T/IR $\Delta \beta$ and R/IR $\Delta \beta$. RMSD traces for each domain of IR, similar to Fig. S6, are shown for TAMD simulations of each complex in Fig. S10. These data are consistent with what is seen in the MD equilibration (vide supra) and in our previous simulations. ${ }^{1}$ We observe that: (i) domains maintain their overall fold; (ii) L1 and L2 domains are relatively rigid with CR domains (connecting L1 and L2) having higher flexibility; (iii) fibronectin domains are most flexible with pronounced flexibility in the F3 domains due to conformational change in each ligand (see Table S2 for mean distances between the pair of F2-F2' and F3-F3' domains).

Similar to Fig. S7, we show traces of collective geometric variables that characterize interdomain flexibility of $\operatorname{IR} \Delta \beta$ during TAMD simulations in Fig. S11. Consistent with what is observed during MD simulations (vide sipra), the F1-F2 hinges of IR $\Delta \beta$ for each complex tend to stay more open than what is observed in the crystal structure, while the L2-F1 hinges on the apex of receptor ectodomain nearly maintain their crystallographic values throughout equilibration. The L1-L2 hinge on the ligand-bound side in the T/IR complex is more open, while the same hinge on the unoccupied side of IR is contracted in comparison to values observed in the crystal structure. The distances between F1-F2 and L1-L2 hinges are near crystallographic for each complex. 
To characterize intersubunit flexibility of IR during TAMD simulations, we show traces of interdomain buried surface areas (BSA) and the size of each binding pocket of IR $\Delta \beta$ in Fig. S12. We observe correlated asymmetric fluctuations in the L1/F2 and L2/F1 interfaces on the ligand-bound side of T/IR complex: the L1/F2 interfaces become more open, and the L2/F1 interfaces become significantly closed, while both interfaces on the unoccupied side in the T/IR complex maintain values either more than or close to the values in the crystal structure. On the ligand-bound side of the R/IR complex, the L1/F2 interface is more compact than the crystal structure, while the L2/F1 interface during early part of TAMD simulation is more open only to recover to crystallographic values in the end. The binding pocket on the ligand-bound side of T/IR does not appreciably change during TAMD simulation, but the binding pocket on the unoccupied side recovers to crystallograhic size. For the R-IR complex, binding pocket on the ligand-bound side of IR $\Delta \beta$ transitions to become more closed than initially, while the pocket on the unoccupied side of IR does not appreciably change in comparison to its initial size. It is interesting to note that closing transition in the ligand-occupied binding pocket of $\mathrm{R} / \mathrm{IR} \Delta \beta$ complex is concurrent with the onset of increased contacts of R-insulin with L1, which occurs after the C-terminal tail (B21-B30) has moved away from the $\mathrm{N}$-terminus of the A-chain exposing the receptor-binding surface of R-insulin (see results in the main manuscript).

These observations collectively are consistent with our previous work, ${ }^{1,6}$ and suggest that ligand-binding only induces subtle conformational changes in the individual domains of each IR subunit. Moreover, the asymmetry observed in the intersubunit interfaces and binding pockets allows IR to work as a "harmonic-oscillator" where small amount of thermal energy can drive the receptor between symmetry-inverted asymmetric active states as proposed by Kiselyov et al. ${ }^{24}$ We also note that these observations are also consistent with a "see-saw" model of negative cooperativity. ${ }^{2}$

Key structural properties characterizing the dynamics of receptor and bound-insulin molecules in the minimum-free energy path computed by the string method. For each image in the MFEP, we show BSA at all four intersubunit interfaces of IR $\Delta \beta$ for each insulin/receptor complex in Fig. S13A. Key BSA and RMSD of bound-insulin molecules, similar to Fig $2 A$ (see main manuscript), are reported in Fig. S13B for each image in MFEP along with snapshots of CT peptide in Fig. S13C. Snapshots for two different views of R-insulin on the L1 surface from the lowest free-energy image 18 are shown in Fig. S14. These observations are consistent with what is observed in TAMD simulations (see main and supplemental results for TAMD simulations).

Equilibration of $R$-insulin docked IR $\beta$ in the displaced-CT model. We equilibrated the R-insulin docked IR $\Delta \beta$ (displaced-CT model; see Fig. 5 in the main manuscript) via a 12-ns explicit-solvent MD simulation. Snapshots from this equilibration run are shown in Fig. S17A and key geometric properties of the receptor and bound-ligand are shown in Fig. S17B. Specifically, we observe that buried surface area between R-insulin and the L1 domain of IR increases during equilibration suggesting an increased registry between hormone and the key ligand-binding surface on L1. We also observe that the receptor pocket to which R-insulin is bound becomes more compact, while the unoccupied pocket of the receptor becomes more open during equilibration suggesting the binding of a single ligand on one-side of IR likely loosens interfaces on the other-side which can allow binding of another ligand molecules with 2:2 (insulin:IR) stoichiometry. Interestingly, we also observe that ligand-binding leads to the closing of the legs of receptor ectodomain as indicated by distance between the pair of FnIII domains.

Additional TAMD simulations. We have carried out two additional sets of TAMD simulations for each insulin/IR complex, and properties of bound-insulin molecules, similar to Fig. $2 A$, are reported in Fig. S19. Similar to as observed for the original 4 40-ns long TAMD simulation of each complex (see main manuscript results), we observe the conformational change in the C-terminus of the B-chain of each insulin (1); Fig. S19), which results in the exposure of the buried surface of each insulin (2); Fig. S19). The CT-peptide maintains its contact surface with L1 (3); Fig. S19) as observed in the original TAMD simulations (see main manuscript).

Comparison of TAMD-generated conformations of each insulin with a novel insulin analogue. We have compared 
TAMD-generated conformations of each insulin with a novel-insulin analogue crystallized by Jiracek et al., ${ }^{25}$ which differs from the wild-type hormone in the conformation of the C-terminus of the B-chain. We have made these comparisons in two different ways. Firstly, we compute from TAMD simulation of each insulin the RMSD of $\mathrm{C}_{\alpha}$ (with reference to the crystal structure of novel analogue; PDB code 2WRX) atoms of the C-terminal tail residues (B21-B30), and show the snapshot of the overlay of lowest-RMSD (in C-tail) conformation of each insulin with the novel analogue in the top panels of Fig. S20. Secondly, we conduct a 10-ns long explicit-solvent MD equilibration of the novel insulin analogue (PDB code 2WRX), and compute RMSD, as in the first step, of the C-terminal tail residues of this analogue with reference to lowest-RMSD conformation of each insulin observed from TAMD simulations. Snapshots from these overlays are reported in the bottom panels of Fig. S20. In all cases, we find that TAMD-generated conformations conform to what has been shown by novel insulin analogue in that the C-terminus of the B-chain moves away from the N-terminal residues of the A-chain. Also, we observe from MD simulations that the C-terminus of the B-chain of novel analogue has considerable flexibility (RMSD of $\mathrm{C}_{\alpha}$ tail residues is $\sim 6.22 \pm 2.55$ $\AA$ ). We note that we have also compared (data not shown) in a similar manner another nearly-complete insulin analogue (PDB code 2WS1) with our TAMD-generated conformations of each insulin and similar RMSD values are seen. These data further lend support to our conclusion that TAMD-generated conformations are statistically signifcant, and are representative of conformations observed in the crystal structures as shown in this work and earlier. $^{14}$

Flexibility of membrane-proximal domains of IR in apo vs. ligand-bound simulations. Finally, it may be informative to understand how the dynamics of soluble ectodomain differs in apo vs. ligand-bound forms because insulin binding to the holo-receptor triggers compaction of ligand/receptor complex ${ }^{26}$ causing trans-autophosphorylation in the cytoplasmic kinase domains. Therefore, we analyzed 18 simulations (6 apo and 12 ligand-bound) of apo vs. ligand-bound forms of IR and IGF1R for distances between the centers of mass of pair of membrane-proximal ("legs") F2-F2' and F3-F3' domains (Table S2). Generally, we find the following for the F3-F3' pair : (i) soluble apoectodomain structures of $\mathrm{IR}^{2,3}$ and IGF1R ${ }^{27}$ display an equal probability of opening/closing of receptor legs (with reference to the starting structure); (ii) the ligand-binding seems to stabilize the legs of soluble ectodomains such that the distances either stay relatively close to the initial structure or tend to decrease by $\sim 3-5 \AA$ on average. The distances of the F2-F2' pair are (nearly) always slightly greater than what is observed in the initial structures, both for apo and ligand-bound trajectories.

\section{SUPPLEMENTAL REFERENCES}

1. Vashisth, H. \& Abrams, C. (2010). Docking of insulin to a structurally equilibrated insulin receptor ectodomain. Proteins 78, 1531-1543.

2. McKern, N., Lawrence, M., Streltsov, V., Lou, M., Adams, T., Lovrecz, G., Elleman, T., Richards, K., Bentley, J., Pilling, P., Hoyne, P., Cartledge, K., Pham, T., Lewis, J., Sankovich, S., Stoichevska, V., Silva, E., Robinson, C., Frenkel, M., Sparrow, L., Fernley, R., Epa, V. \& Ward, C. (2006). Structure of the insulin receptor ectodomain reveals a folded-over conformation. Nature 443, 218-221.

3. Smith, B., Huang, K., Kong, G., Chan, S., Nakagawa, S., Menting, J., Hu, S., Whittaker, J., Steiner, D., Katsoyannis, P., Ward, C., Weiss, M. \& Lawrence, M. (2010). Structural resolution of a tandem hormone-binding element in the insulin receptor and its implications for design of peptide agonists. Proc. Natl. Acad. Sci. USA 107, 6771-6776.

4. Humphrey, W., Dalke, A. \& Schulten, K. (1996). VMD - Visual Molecular Dynamics. J. Mol. Graph. 14, 33-38.

5. Vashisth, H. (2010). Ph.D. thesis, Drexel University.

6. Vashisth, H. \& Abrams, C. (2010). All-atom structural models for complexes of insulin-like growth factors IGF1 and IGF2 with their cognate receptor. J. Mol. Biol. 400, 645-658.

7. Phillips, J., Braun, R., Wang, W., Gumbart, J., Tajkhorshid, E., Villa, E., Chipot, C., Skeel, R., Kalé, L. \& Schulten, K. (2005). Scalable molecular dynamics with NAMD. J. Comput. Chem. 26, 1781-1802.

8. MacKerell, Jr., A.D., Bashford, D., Bellott, M., Dunbrack, R.L., Jr., Evanseck, J.D., Field, M.J., Fischer, S., Gao, J., Guo, H., Ha, S., Joseph-McCarthy, D., Kuchnir, L., Kuczera, K., Lau, F.K., Mattos, C., Michnick, S., Ngo, T., Nguyen, D.T., Prodhom, B., Reiher III, W.E., Roux, B., Schlenkrich, M., Smith, J.C., Stote, R., Straub, J., Watanabe, M., Wiórkiewicz-Kuczera, J., Yin, D. \& 
Karplus, M. (1998). All-atom empirical potential for molecular modeling and dynamics studies of proteins. J. Phys. Chem. B 102, 3586-3616.

9. MacKerell, Jr., A.D., Feig, M. \& Brooks, C. L., III (2004). Extending the treatment of backbone energetics in protein force fields: limitations of gas-phase quantum mechanics in reproducing protein conformational distributions in molecular dynamics simulations. J. Comput. Chem. 25, 1400-1415.

10. Maragliano, L. \& Vanden-Eijnden, E. (2006). A temperature accelerated method for sampling free energy and determining reaction pathways in rare events simulations. Chem. Phys. Lett. 426, 168-175.

11. Maragliano, L. \& Vanden-Eijnden, E. (2008). Single-sweep methods for free energy calculations. J. Chem. Phys. 128, 184110.

12. Vanden-Eijnden, E. (2009). Some Recent Techniques for Free Energy Calculations. J. Comput. Chem. 30, 1737-1747.

13. Abrams, C.F. \& Vanden-Eijnden, E. (2010). Large-scale conformational sampling of proteins using temperature-accelerated molecular dynamics. Proc. Natl. Acad. Sci. USA 107, 4961-4966.

14. Vashisth, H., Maragliano, L. \& Abrams, C.F. (2012). "DFG-flip" in the insulin receptor kinase is facilitated by a helical intermediate state of the activation loop. Biophys. J. 102, 1979-1987.

15. E, W.N., Ren, W.Q. \& Vanden-Eijnden, E. (2002). String method for the study of rare events. Phys. Rev. B 66, 052301.

16. Weinan, E., Ren, W.Q. \& Vanden-Eijnden, E. (2005). Finite temperature string method for the study of rare events. J. Phys. Chem. B 109, 6688-6693.

17. Ren, W., Vanden-Eijnden, E., Maragakis, P. \& E, W.N. (2005). Transition pathways in complex systems: Application of the finite-temperature string method to the alanine dipeptide. J. Chem. Phys. 123, 134109.

18. E, W.N., Ren, W.Q. \& Vanden-Eijnden, E. (2007). Simplified and improved string method for computing the minimum energy paths in barrier-crossing events. J. Chem. Phys. 126, 164103.

19. Maragliano, L., Fischer, A., Vanden-Eijnden, E. \& Ciccotti, G. (2006). String method in collective variables: Minimum free energy paths and isocommittor surfaces. J. Chem. Phys. 125, 024106.

20. Vanden-Eijnden, E. \& Venturoli, M. (2009). Revisiting the finite temperature string method for the calculation of reaction tubes and free energies. J. Chem. Phys. 130, 194103.

21. Miller, T.F., Vanden-Eijnden, E. \& Chandler, D. (2007). Solvent coarse-graining and the string method applied to the hydrophobic collapse of a hydrated chain. Proc. Natl. Acad. Sci. USA 104, 14559-14564.

22. Zhu, F.Q. \& Hummer, G. (2010). Pore opening and closing of a pentameric ligand-gated ion channel. Proc. Natl. Acad. Sci. USA 107, 19814-19819.

23. Gan, W., Yang, S. \& Roux, B. (2009). Atomistic View of the Conformational Activation of Src Kinase Using the String Method with Swarms-of-Trajectories. Biophys. J. 97, L8-L10.

24. Kiselyov, V., Versteyhe, S., Gauguin, L. \& De Meyts, P. (2009). Harmonic oscillator model of the insulin and IGF1 receptors' allosteric binding and activation. Mol. Syst. Biol. 5, 1-12.

25. Jiracek, J., Zakova, L., Antolikova, E., Watson, C.J., Turkenburg, J.P., Dodson, G.G. \& Brzozowski, A.M. (2010). Implications for the active form of human insulin based on the structural convergence of highly active hormone analogues. Proc. Natl. Acad. Sci. USA 107, 1966-1970.

26. Flörke, R., Schnaith, K., Passlack, W., Wichert, M., Kuehn, L., Fabry, M., Federwisch, M. \& Reinauer, H. (2001). Hormonetriggered conformational changes within the insulin-receptor ectodomain: requirement for transmembrane anchors. Biochem. J. 360, 189-198.

27. Whitten, A., Smith, B., Menting, J., Margetts, M., McKern, N., Lovrecz, G., Adams, T., Richards, K., Bentley, J., Trewhella, J., Ward, C. \& Lawrence, M. (2009). Solution structure of ectodomains of the insulin receptor family: the ectodomain of the Type 1 insulin-like growth factor receptor displays asymmetry of ligand binding accompanied by limited conformational change. J. Mol. Biol. 394, 878-892.

28. Pullen, R., Lindsay, D., Wood, S., Tickle, I., Blundell, T., Wollmer, A., Krail, G., Brandenburg, D., Zahn, H., Gliemann, J. \& Gammeltoft, S. (1976). Receptor-binding region of insulin. Nature 259, 369-373.

29. De Meyts, P., Obberghen, E., Roth, J., Wollmer, A. \& Brandenburg, D. (1978). Mapping of the residues responsible for the negative cooperativity of the receptor-binding region of insulin. Nature 273, 504-509.

30. Kristensen, C., Kjeldsen, T., Wiberg, F., Schäffer, L., Hach, M., Havelund, S., Bass, J., Steiner, D. \& Andersen, A. (1997). Alanine scanning mutagenesis of insulin. J. Biol. Chem. 272, 12978-12983.

31. Chen, H., Shi, M., Guo, Z., Tang, Y., Qiao, Z., Liang, Z. \& Feng, Y. (2000). Four new monomeric insulins obtained by alanine scanning the dimer-forming surface of the insulin molecule. Protein Eng. 13, 779-782.

32. De Meyts, P. \& Whittaker, J. (2002). Structural biology of insulin and IGF1 receptors: implications for drug design. Nat. Rev. Drug. Discov. 1, 769-783.

33. De Meyts, P. (2004). Insulin and its receptor: structure, function and evolution. BioEssays 26, 1351-1362. 
34. De Meyts, P. (2008). The insulin receptor: a prototype for dimeric, allosteric membrane receptors? Trends Biochem. Sci. 33, 376-384.

35. Nakamura, T., Takahashi, H., Takahashi, M., Shimba, N., Suzuki, E.i. \& Shimada, I. (2010). Direct determination of the insulin-insulin receptor interface using transferred cross-saturation experiments. J. Med. Chem. 53, 1917-1922.

36. Williams, P., Mynarcik, D., Yu, G. \& Whittaker, J. (1995). Mapping of an $\mathrm{NH}_{2}$-terminal ligand binding site of the insulin receptor by alanine scanning mutagenesis. J. Biol. Chem. 270, 3012-3016.

37. Whittaker, J. \& Whittaker, L. (2005). Characterization of the functional insulin binding epitopes of the full-length insulin receptor. J. Biol. Chem. 280, 20932-20936.

38. Mynarcik, D., Williams, P., Schaffer, L., Yu, G. \& Whittaker, J. (1997). Analog binding properties of insulin receptor mutants. J. Biol. Chem. 272, 2077-2081.

39. Schaefer, E., Siddle, K. \& Ellis, L. (1990). Deletion analysis of the human insulin receptor ectodomain reveals independently folded soluble subdomains and insulin binding by a monomeric $\alpha$-subunit. J. Biol. Chem. 265, 13248-13253.

40. Kristensen, C., Andersen, A., Hach, M., Wiberg, F., Schäffer, L. \& Kjeldsen, T. (1995). A single-chain insulin-like growth factor I/insulin hybrid binds with high affinity to the insulin receptor. Biochem. J. 305, 981-986.

41. Schlein, M., Havelund, S., Kristensen, C., Dunn, M. \& Kaarsholm, N. (2000). Ligand-induced conformational change in the minimized insulin receptor. J. Mol. Biol. 303, 161-169.

42. Brandt, J., Andersen, A. \& Kristensen, C. (2001). Dimeric fragment of the insulin receptor $\alpha$-subunit binds insulin with full holoreceptor affinity. J. Biol. Chem. 276, 12378-12384.

43. Surinya, K., Molina, L., Soos, M., Brandt, J., Kristensen, C. \& Siddle, K. (2002). Role of insulin receptor dimerization domains in ligand binding, cooperativity, and modulation by anti-receptor antibodies. J. Biol. Chem. 277, 16718-16725.

44. Kristensen, C., Andersen, A., Østergaard, S., Hansen, P. \& Brandt, J. (2002). Functional reconstitution of insulin receptor binding site from non-binding receptor fragments. J. Biol. Chem. 277, 18340-18345.

45. Kurose, T., Pashmforoush, M., Yoshimasa, Y., Carroll, R., Schwartz, G., Burke, G., Katsoyannis, P. \& Steiner, D. (1994). Crosslinking of a B25 Azidophenylalanine insulin derivative to the carboxyl-terminal regions of the $\alpha$-subunit of the insulin receptor. J. Biol. Chem. 269, 29190-29197.

46. Mynarcik, D., Yu, G. \& Whittaker, J. (1996). Alanine-scanning mutagenesis of a C-terminal ligand binding domain in the insulin receptor $\alpha$ subunit. J. Biol. Chem. 271, 2439-2442.

47. Kristensen, C., Wiberg, F. \& Andersen, A. (1999). Specificity of insulin and insulin-like growth factor I receptors investigated using chimeric mini-receptors. J. Biol. Chem. 274, 37351-37356.

48. Molina, L., Marino-Buslje, C., Quinn, D. \& Siddle, K. (2000). Structural domains of the insulin receptor and IGF receptor required for dimerization and ligand binding. FEBS Lett. 467, 226-230.

49. Whittaker, L., Hao, C., Fu, W. \& Whittaker, J. (2008). High-affinity insulin binding: insulin interacts with two receptor ligand binding sites. Biochemistry 47, 12900-12909.

50. Ward, C., Lawrence, M., Streltsov, V., Adams, T. \& McKern, N. (2007). The insulin and EGF receptor structures: new insights into ligand-induced receptor activation. Trends Biochem. Sci. 32, 129-137.

51. Lawrence, M., McKern, N. \& Ward, C. (2007). Insulin receptor structure and its implications for the IGF-1 receptor. Curr. Opin. Struct. Biol. 17, 699-705.

52. Zhang, B. \& Roth, R. (1991). A region of the insulin receptor important for ligand binding (residues 450-601) is recognized by patients' autoimmune antibodies and inhibitory monoclonal antibodies. Proc. Natl. Acad. Sci. USA 88, 9858-9862.

53. Fabry, M., Schaefer, E., Ellis, L., Kojro, E., Fahrenholz, F. \& Brandenburg, D. (1992). Detection of a new hormone contact site within the insulin receptor ectodomain by the use of a novel photoreactive insulin. J. Biol. Chem. 267, 8950-8956.

54. Schumacher, R., Soos, M., Schlessinger, J., Brandenburg, D., Siddle, K. \& Ullrich, A. (1993). Signaling-competent receptor chimeras allow mapping of major insulin receptor binding domain determinants. J. Biol. Chem. 268, 1087-1094.

55. Hao, C., Whittaker, L. \& Whittaker, J. (2006). Characterization of a second ligand binding site of the insulin receptor. Biochem. Biophys. Res. Commun. 347, 334:339.

56. Benyoucef, S., Surinya, K., Hadaschik, D. \& Siddle, K. (2007). Characterization of insulin/IGF hybrid receptors: contributions of the insulin receptor L2 and Fn1 domains and the alternatively spliced exon 11 sequence to ligand binding and receptor activation. Biochem. J. 403, 603-613.

57. Wan, Z.L., Huang, K., Xu, B., Hu, S.Q., Wang, S.H., Chu, Y.C., Katsoyannis, P.G. \& Weiss, M.A. (2005). Diabetes-associated mutations in human insulin: Crystal structure and photo-cross-linking studies of A-chain variant insulin Wakayama. Biochemistry 44, 5000-5016.

58. Shoelson, S., Fickova, M., Haneda, M., Nahum, A., Musso, G., Kaiser, E.T., Rubenstein, A.H. \& Tager, H. (1983). Identification of a mutant human insulin predicted to contain a serine-for-phenylalanine substitution. Proc. Natl. Acad. Sci. USA 80, 7390- 
7394.

59. Shoelson, S., Haneda, M., Blix, P., Nanjo, A., Sanke, T., Inouye, K., Steiner, D., Rubenstein, A. \& Tager, H. (1983). Three mutant insulins in man. Nature 302, 540-543.

60. Nanjo, K., Sanke, T., Miyano, M., Okai, K., Sowa, R., Kondo, M., Nishimura, S., Iwo, K., Miyamura, K., Given, B.D., Chan, S.J., Tager, H.S., Steiner, D.F. \& Rubenstein, A.H. (1986). Diabetes due to secretion of a structurally abnormal insulin (insulin Wakayama). Clinical and functional characteristics of LeuA3 insulin. J. Clin. Invest. 77, 514-519.

61. Carroll, R.J., Hammer, R.E., Chan, S.J., Swift, H.H., Rubenstein, A.H. \& Steiner, D.F. (1988). A mutant human proinsulin is secreted from islets of Langerhans in increased amounts via an unregulated pathway. Proc. Natl. Acad. Sci. USA 85, 8943-8947. 
TABLE S1: Key site-1 and site-2 residues of insulin ${ }^{28-35}$ and the receptor ${ }^{36-56}$ suggested by site-specific mutagenesis and chimeric receptor constructs. Red indicates sites of clinical insulin mutations: ${ }^{33,57} \mathrm{Phe}^{\mathrm{B} 24} \rightarrow \mathrm{Ser}^{, 58}$

$\mathrm{Phe}^{\mathrm{B} 25} \rightarrow \mathrm{Leu}^{59} \mathrm{Val}^{\mathrm{A} 3} \rightarrow \mathrm{Leu}^{60}$ and His ${ }^{\mathrm{B} 10} \rightarrow \mathrm{Asp}^{61}{ }^{61}$ The domains of the receptor to which each residue of IR belongs are also listed.

\begin{tabular}{|c|c|c|c|c|c|}
\hline \multicolumn{2}{|c|}{ Insulin } & \multicolumn{4}{|c|}{ Receptor } \\
\hline \multirow{2}{*}{$\frac{\text { site-1 }}{\text { Gly }^{\mathrm{A} 1}}$} & \multirow{2}{*}{$\frac{\text { site-2 }}{\text { Ser }^{\mathrm{A} 12}}$} & \multicolumn{2}{|c|}{ site-1 } & \multicolumn{2}{|c|}{ site-2 } \\
\hline & & $\mathrm{Asp}^{12}$ & (L1) & Lys $^{484}$ & (F1) \\
\hline $\mathrm{Ile}^{\mathrm{A} 2}$ & $\mathrm{Leu}^{\mathrm{A} 13}$ & $\mathrm{Ile}^{13}$ & (L1) & $\mathrm{Leu}^{552}$ & (F1) \\
\hline $\mathrm{Val}^{\mathrm{A} 3 a}$ & $\mathrm{Tyr}^{\mathrm{A} 14}$ & $\operatorname{Arg}^{14}$ & (L1) & $\mathrm{Asp}^{591}$ & (F1) \\
\hline $\mathrm{G} \ln ^{\mathrm{A} 5}$ & $\mathrm{Glu}^{\mathrm{A} 17}$ & $\mathrm{Asn}^{15}$ & (L1) & $\mathrm{Ile}^{602}$ & (F2) \\
\hline $\mathrm{Thr}^{\mathrm{A} 8}$ & $\mathrm{Leu}^{\mathrm{B} 6}$ & $\mathrm{Gln}^{34}$ & (L1) & Lys $^{616}$ & (F2) \\
\hline $\operatorname{Tyr}^{\mathrm{A} 19}$ & $\mathrm{His}^{\mathrm{B} 10}$ & $\mathrm{Leu}^{36}$ & (L1) & $\mathrm{Asp}^{620}$ & (F2) \\
\hline $\mathrm{Asn}^{\mathrm{A} 21}$ & $\mathrm{Glu}^{\mathrm{B} 13}$ & $\mathrm{Leu}^{37}$ & (L1) & Pro $^{621}$ & (F2) \\
\hline $\mathrm{Val}^{\mathrm{B} 12}$ & $\mathrm{Leu}^{\mathrm{B} 17}$ & $\mathrm{Phe}^{39}$ & (L1) & & \\
\hline $\mathrm{Tyr}^{\mathrm{B} 16}$ & $\mathrm{Val}^{\mathrm{B} 18}$ & $\mathrm{Glu}^{44}$ & (L1) & & \\
\hline $\mathrm{Phe}^{\mathrm{B} 24 b}$ & & $\mathrm{Phe}^{64}$ & (L1) & & \\
\hline $\mathrm{Phe}^{\mathrm{B} 25 c}$ & & $\operatorname{Tyr}^{67}$ & (L1) & & \\
\hline $\mathrm{Tyr}^{\mathrm{B} 26}$ & & $\mathrm{Phe}^{89}$ & (L1) & & \\
\hline & & $\mathrm{Asn}^{90}$ & (L1) & & \\
\hline & & Tyr $^{91}$ & (L1) & & \\
\hline & & $\mathrm{Phe}^{705}$ & (CT) & & \\
\hline & & $\mathrm{Glu}^{706}$ & $(\mathrm{CT})$ & & \\
\hline & & $\mathrm{Asp}^{707}$ & (CT) & & \\
\hline & & $\mathrm{Tyr}^{708}$ & $(\mathrm{CT})$ & & \\
\hline & & $\mathrm{Leu}^{709}$ & $(\mathrm{CT})$ & & \\
\hline & & $\mathrm{Asn}^{711}$ & $(\mathrm{CT})$ & & \\
\hline & & $\mathrm{Val}^{712}$ & $(\mathrm{CT})$ & & \\
\hline & & $\mathrm{Phe}^{714}$ & $(\mathrm{CT})$ & & \\
\hline & & $\operatorname{Pro}^{716}$ & $(\mathrm{CT})$ & & \\
\hline & & $\operatorname{Arg}^{717}$ & $(\mathrm{CT})$ & & \\
\hline
\end{tabular}

${ }^{a}$ insulin Wakayama

$b_{\text {insulin Los Angeles }}$

${ }^{c}$ insulin Chicago 
TABLE S2: Separation of legs in the apo and ligand-bound simulations of the receptors of the insulin family. Mean $(\mu)$ and standard deviation $(\sigma)$ of distances between the centers of mass (COM) of the pair of F3/F3' and F2/F2' domains are reported. Arrows indicate the increase/decrease of mean separation (in comparison to their base-values) between the pair of fibronectin domains.

\begin{tabular}{|c|c|c|c|c|c|}
\hline System & Initial coordinates (ref.) & $\begin{array}{l}\text { Simulation } \\
\text { type (ref.) }\end{array}$ & $\begin{array}{c}\text { Trajectory } \\
\text { length }(\sim \mathrm{ns})\end{array}$ & $\begin{array}{l}\mathrm{F}_{\text {com }}-\mathrm{F}_{\text {com }}^{\prime}(\AA) \\
\left.\mu \pm \sigma \text { (base-value }^{*}\right)\end{array}$ & $\begin{array}{l}\mathrm{F} 2_{\text {com }}-\mathrm{F} 2_{\text {com }}^{\prime}(\AA) \\
\left.\mu \pm \sigma \text { (base-value }^{*}\right)\end{array}$ \\
\hline apo-IR $\Delta \beta \mathrm{w} / \mathrm{o}$ CT & $2 \mathrm{DTG}^{2}$ & $\mathrm{MD}^{1}$ & 67 & $111.06 \pm 7.67(102.76) \uparrow$ & $84.34 \pm 6.16(78.68) \uparrow$ \\
\hline apo-IR $\Delta \beta \mathrm{w} / \mathrm{o}$ CT & $2 \mathrm{DTG}^{2}$ & $\mathrm{MD}^{1}$ & 15 & $97.46 \pm 3.43(102.76) \downarrow$ & $79.70 \pm 1.55(78.68) \uparrow$ \\
\hline apo-IR $\Delta \beta \mathrm{w}$ CT & $3 \mathrm{LOH}^{3}$ & $\mathrm{MD}^{\dagger}$ & 28 & $127.62 \pm 11.92(102.76) \uparrow$ & $89.36 \pm 4.98(78.68) \uparrow$ \\
\hline apo-IR $\Delta \beta \mathrm{w}$ CT & $3 \mathrm{LOH}^{3}$ & $\mathrm{MD}^{\dagger}$ & 22 & $99.30 \pm 2.01(102.76) \downarrow$ & $78.96 \pm 1.81(78.68) \uparrow$ \\
\hline apo-IGF1R $\Delta \beta \mathrm{w} / \mathrm{o}$ CT & Homology modeled ${ }^{27}$ & $\mathrm{MD}^{6}$ & 30 & $82.30 \pm 6.16 \quad(93.94) \downarrow$ & $73.03 \pm 2.58(73.66) \downarrow$ \\
\hline apo-IGF1R $\Delta \beta \mathrm{w} / \mathrm{o}$ CT & Homology modeled ${ }^{27}$ & $\mathrm{MD}^{6}$ & 30 & $106.68 \pm 4.74 \quad(93.94) \uparrow$ & $84.42 \pm 3.41(73.66) \uparrow$ \\
\hline $\mathrm{T} / \mathrm{IR} \Delta \beta \mathrm{w} / \mathrm{o} C \mathrm{~T}$ & MC Docking ${ }^{1}$ & $\mathrm{MD}^{1}$ & 30 & $101.27 \pm 3.41(102.76) \downarrow$ & $78.77 \pm 2.28(78.68) \uparrow$ \\
\hline $\mathrm{R} / \mathrm{IR} \Delta \beta \mathrm{w} / \mathrm{o} C \mathrm{~T}$ & MC Docking ${ }^{1}$ & $\mathrm{MD}^{1}$ & 30 & $99.87 \pm 2.71(102.76) \downarrow$ & $79.23 \pm 2.56(78.68) \uparrow$ \\
\hline $\mathrm{IGF} 1 / \mathrm{IGF} 1 \mathrm{R} \Delta \beta \mathrm{w} / \mathrm{o} \mathrm{CT}$ & MC Docking ${ }^{6}$ & $\mathrm{MD}^{6}$ & 36 & $93.36 \pm 3.00 \quad(93.94) \downarrow$ & $80.78 \pm 2.64(73.66) \uparrow$ \\
\hline $\mathrm{IGF} 2 / \mathrm{IGF} 1 \mathrm{R} \Delta \beta \mathrm{w} / \mathrm{o} \mathrm{CT}$ & MC Docking ${ }^{6}$ & $\mathrm{MD}^{6}$ & 36 & $86.39 \pm 2.65 \quad(93.94) \downarrow$ & $74.84 \pm 2.20(73.66) \uparrow$ \\
\hline $\mathrm{T} / \mathrm{IR} \Delta \beta \mathrm{w} \mathrm{CT}$ & MC Docking ${ }^{\ddagger}$ & $\mathrm{MD}^{\ddagger}$ & 10 & $103.59 \pm 1.40(102.76) \uparrow$ & $79.61 \pm 1.50(78.68) \uparrow$ \\
\hline $\mathrm{R} / \mathrm{IR} \Delta \beta \mathrm{w} \mathrm{CT}$ & MC Docking ${ }^{\ddagger}$ & $\mathrm{MD}^{\ddagger}$ & 10 & $100.18 \pm 1.60(102.76) \downarrow$ & $79.15 \pm 1.14(78.68) \uparrow$ \\
\hline $\mathrm{T} / \mathrm{IR} \Delta \beta \mathrm{w} \mathrm{CT}$ & MC Docking ${ }^{\ddagger}$ & TAMD $^{\ddagger}$ & 40 & $110.62 \pm 5.02(102.76) \uparrow$ & $84.27 \pm 3.39(78.68) \uparrow$ \\
\hline $\mathrm{R} / \mathrm{IR} \Delta \beta \mathrm{w} \mathrm{CT}$ & MC Docking ${ }^{\ddagger}$ & $\mathrm{TAMD}^{\ddagger}$ & 40 & $107.95 \pm 5.99(102.76) \uparrow$ & $82.65 \pm 3.28(78.68) \uparrow$ \\
\hline $\mathrm{T} / \mathrm{IR} \Delta \beta \mathrm{w} \mathrm{CT}$ & MC Docking ${ }^{\ddagger}$ & TAMD $^{\ddagger}$ & 28 & $95.22 \pm 3.89(102.76) \downarrow$ & $77.34 \pm 4.08(78.68) \downarrow$ \\
\hline $\mathrm{R} / \mathrm{IR} \Delta \beta \mathrm{w} \mathrm{CT}$ & MC Docking ${ }^{\ddagger}$ & TAMD $^{\ddagger}$ & 31 & $105.55 \pm 3.92(102.76) \uparrow$ & $85.06 \pm 2.94(78.68) \uparrow$ \\
\hline $\mathrm{T} / \mathrm{IR} \Delta \beta \mathrm{w} \mathrm{CT}$ & MC Docking ${ }^{\ddagger}$ & TAMD & 23 & $113.80 \pm 5.63(102.76) \uparrow$ & $86.99 \pm 2.46(78.68) \uparrow$ \\
\hline $\mathrm{R} / \mathrm{IR} \Delta \beta \mathrm{w} \mathrm{CT}$ & MC Docking ${ }^{\ddagger}$ & TAMD $^{\ddagger}$ & 24 & $108.17 \pm 3.84(102.76) \uparrow$ & $86.07 \pm 3.37(78.68) \uparrow$ \\
\hline
\end{tabular}


A

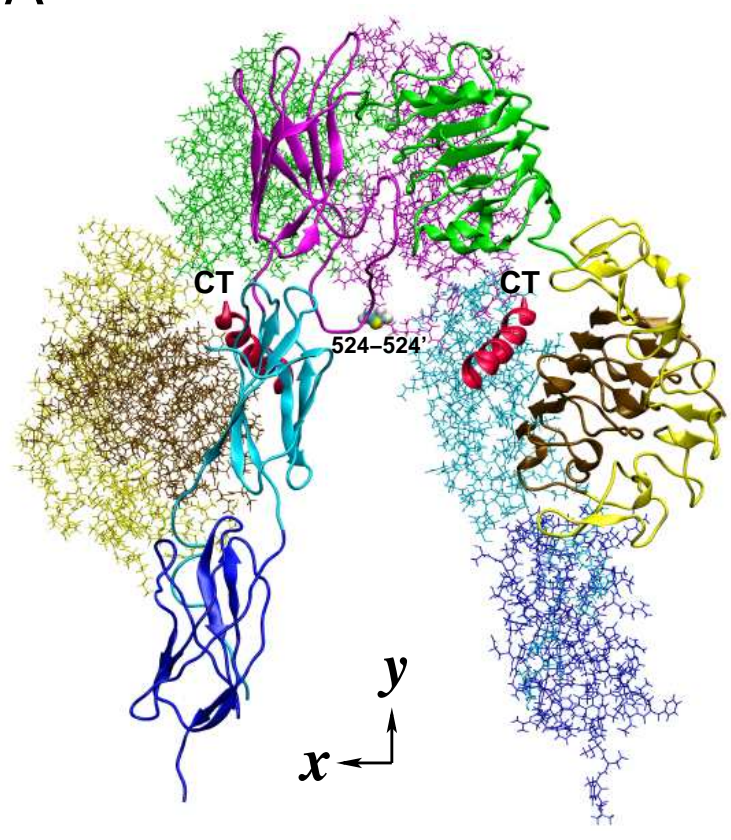

B

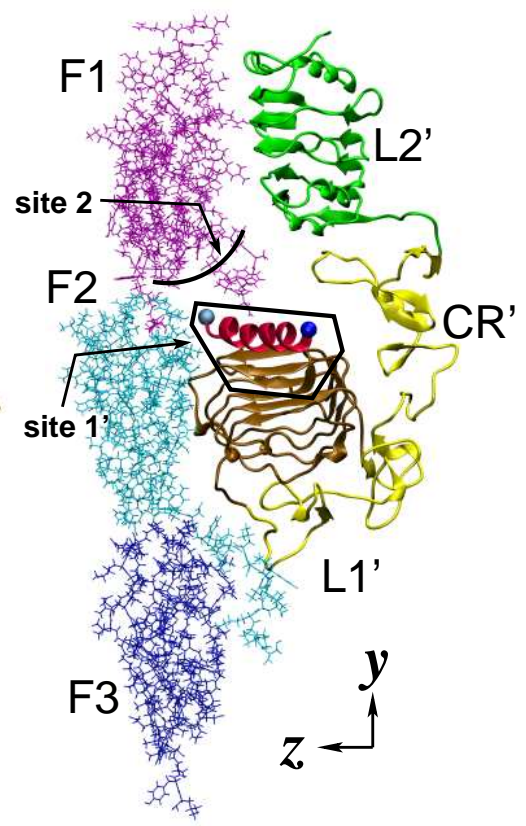

FIGURE S1: $(A)$ Schematic view of the refined IR $\Delta \beta$ crystal structure with CT peptide (PDB code 3LOH) along $+Z$ direction with subunit 1 in cartoon and subunit 2 as bonds. (B) F1, F2, and F3 domains of subunit 1, and L1', CR', and L2' domains of subunit 2 viewed along the -X direction. Domains are denoted in color: brown, L1; yellow, CR; green, L2; magenta, F1; cyan, F2; blue, F3. The intersubunit $\mathrm{Cys}^{524}$-Cys $^{524^{\prime}}$ disulfide is shown in $(A)$ and approximate locations of site $1^{\prime}$ and site 2 indicated in $(B)$. The resolved helical CT peptide in both panels is shown as red cartoon. 

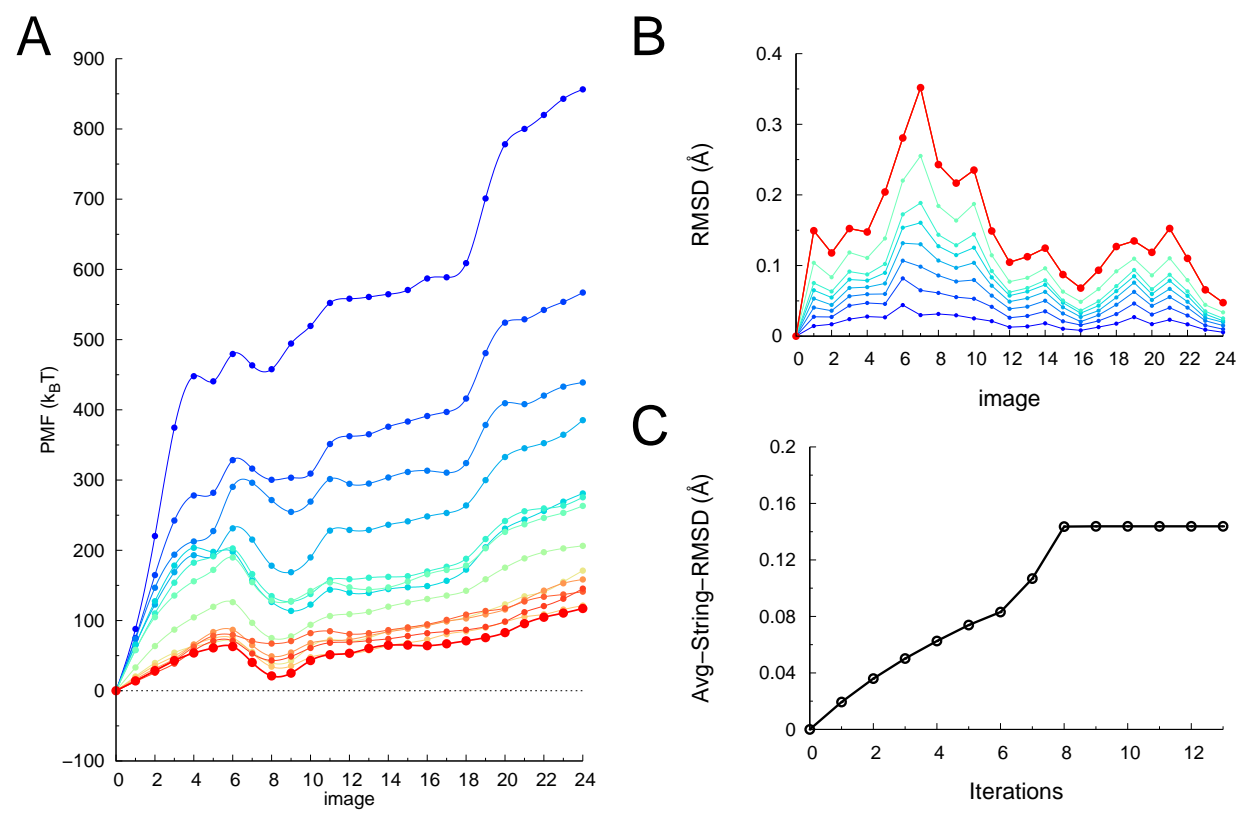

FIGURE S2: Convergence properties of the TAMD-generated activation pathway for T-insulin during each string iteration. (A) Potential of mean force (PMF) profiles. (B) Each image's root-mean squared deviation (RMSD) from respective image in the initial string. (C) RMSD averaged over all images of the string.
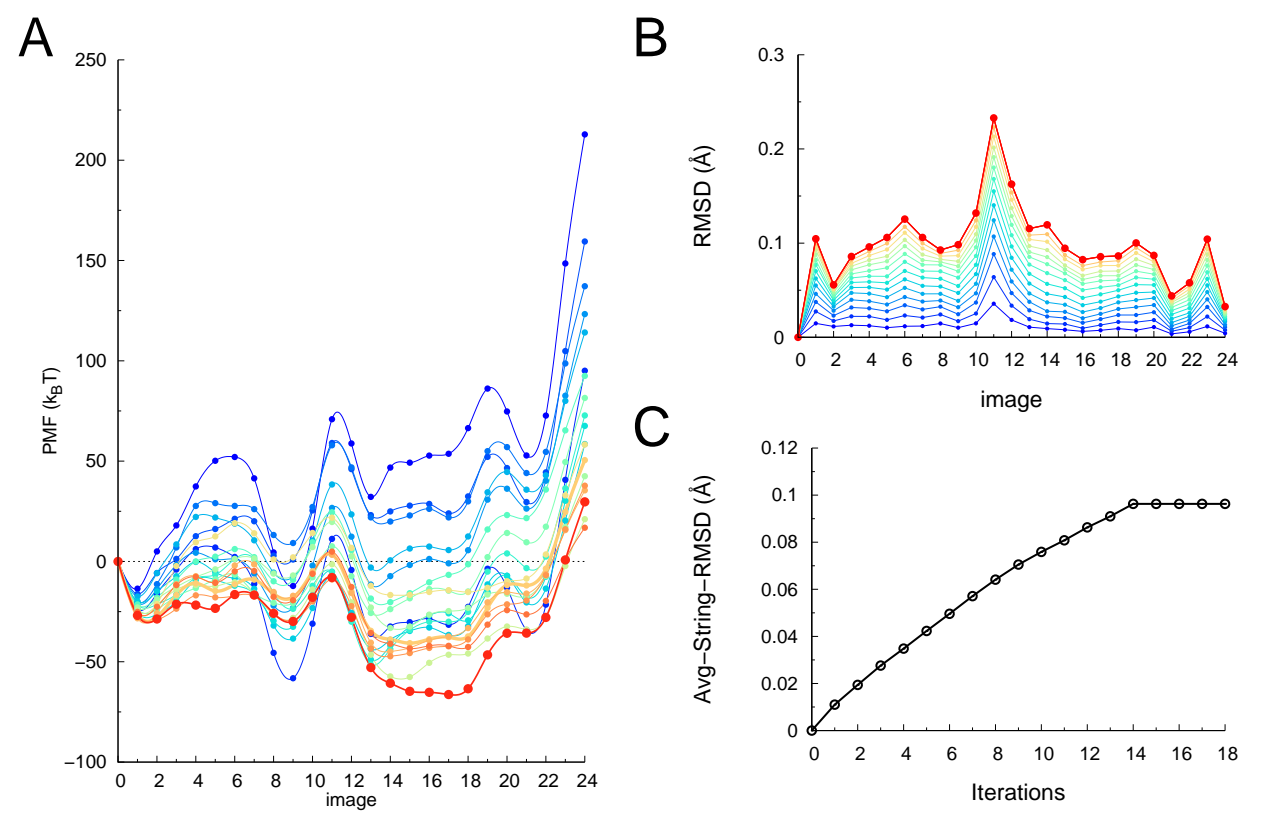

FIGURE S3: Convergence properties of the TAMD-generated activation pathway for R-insulin during each string iteration. (A) Potential of mean force (PMF) profiles. (B) Each image's root-mean squared deviation (RMSD) from respective image in the initial string. (C) RMSD averaged over all images of the string. 

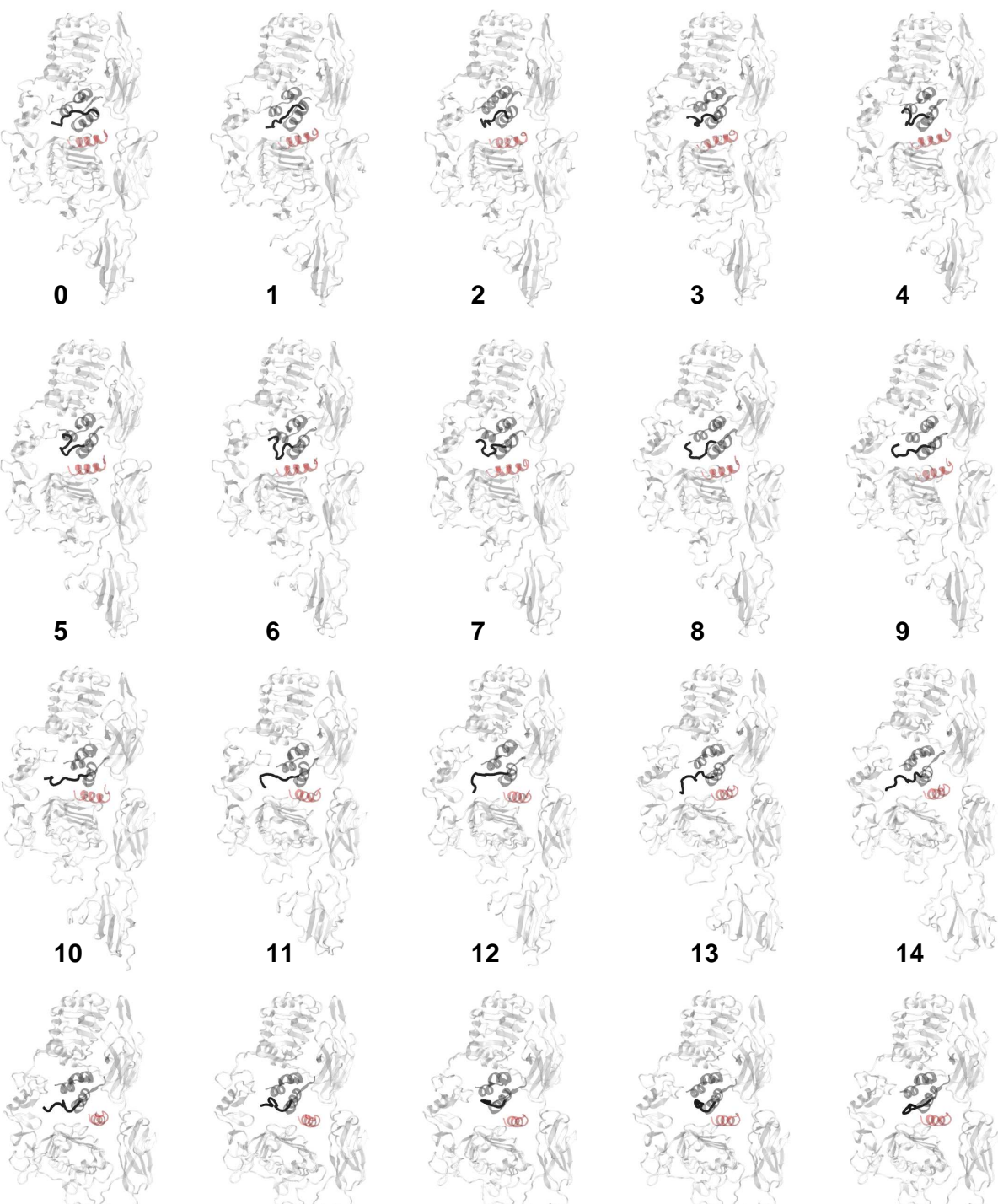

14

15

16
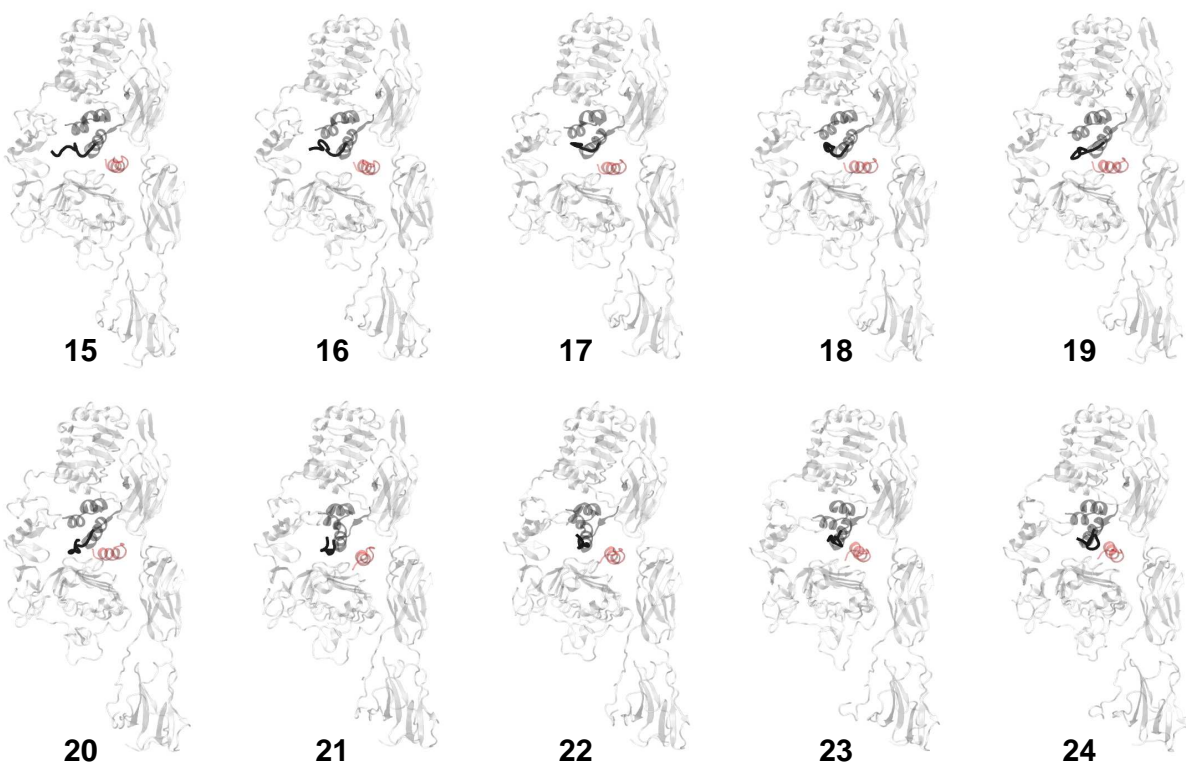

FIGURE S4: Representative conformations of T-insulin docked IR $\Delta \beta$ from 25 minimum-free energy path (MFEP) images, with CT peptide in red transparent cartoon. The insulin molecule is shown in black transparent cartoon except the C-terminus of the B-chain (residue 22-30), which is not transparent. One half of the homo-dimeric receptor is shown as white transparent cartoon. 

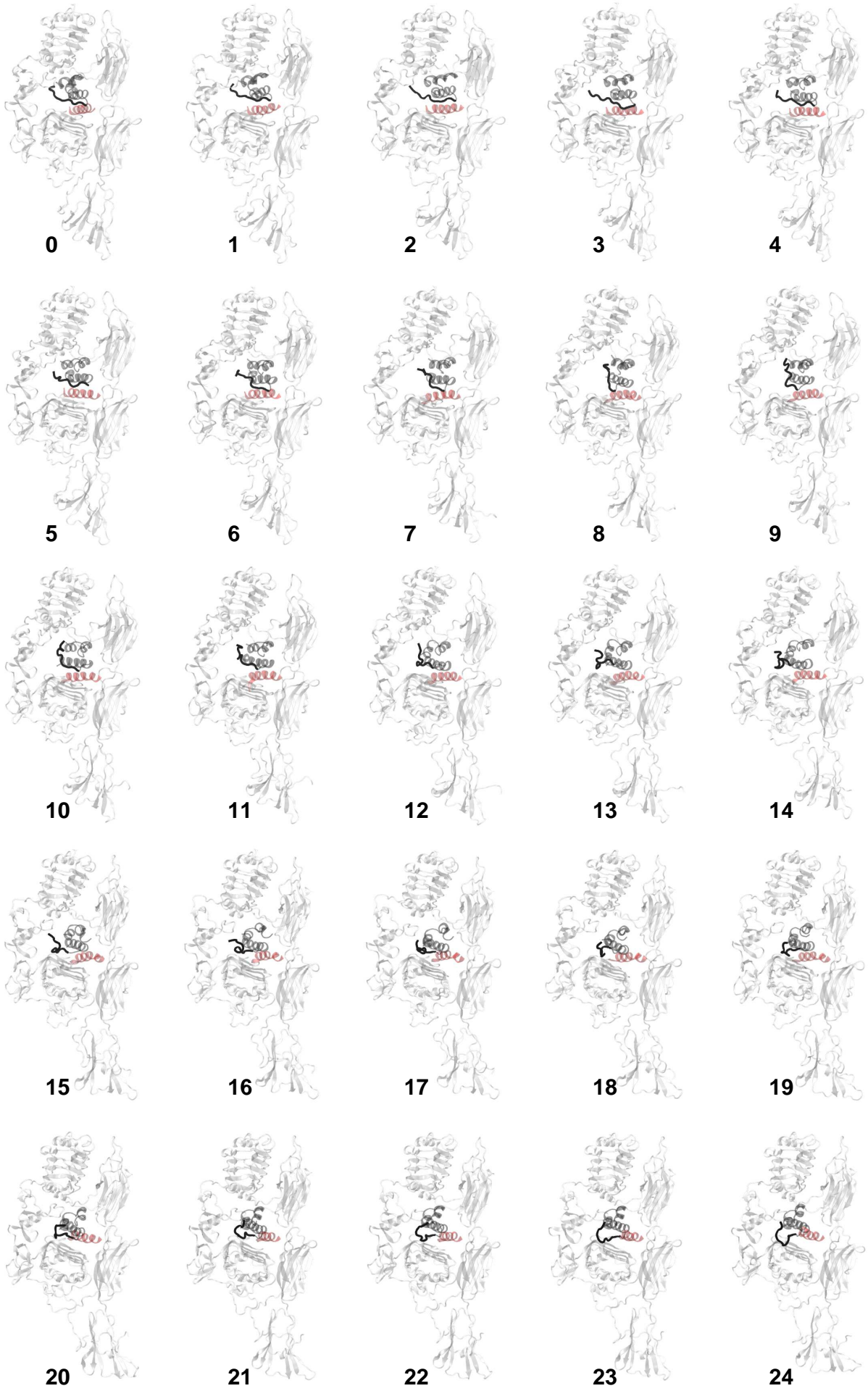

FIGURE S5: Representative conformations of R-insulin docked IR $\Delta \beta$ from 25 minimum-free energy path (MFEP) images, with CT peptide in red transparent cartoon. The insulin molecule is shown in black transparent cartoon except the C-terminus of the B-chain (residue 22-30), which is not transparent. One half of the homo-dimeric receptor is shown as white transparent cartoon. 


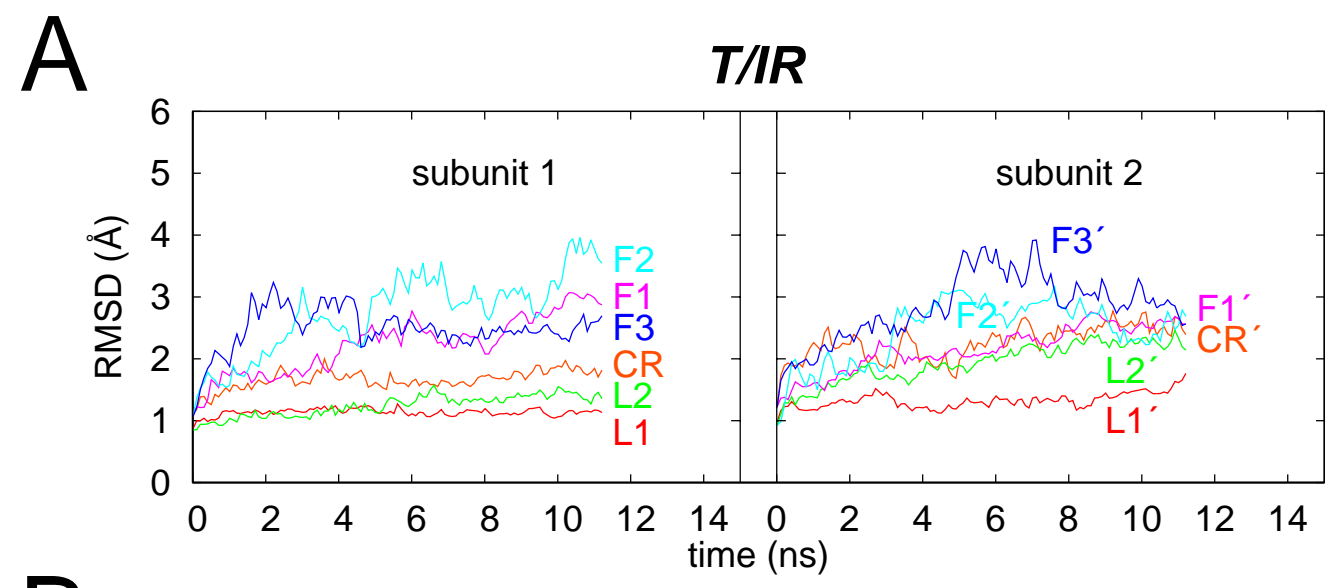

B

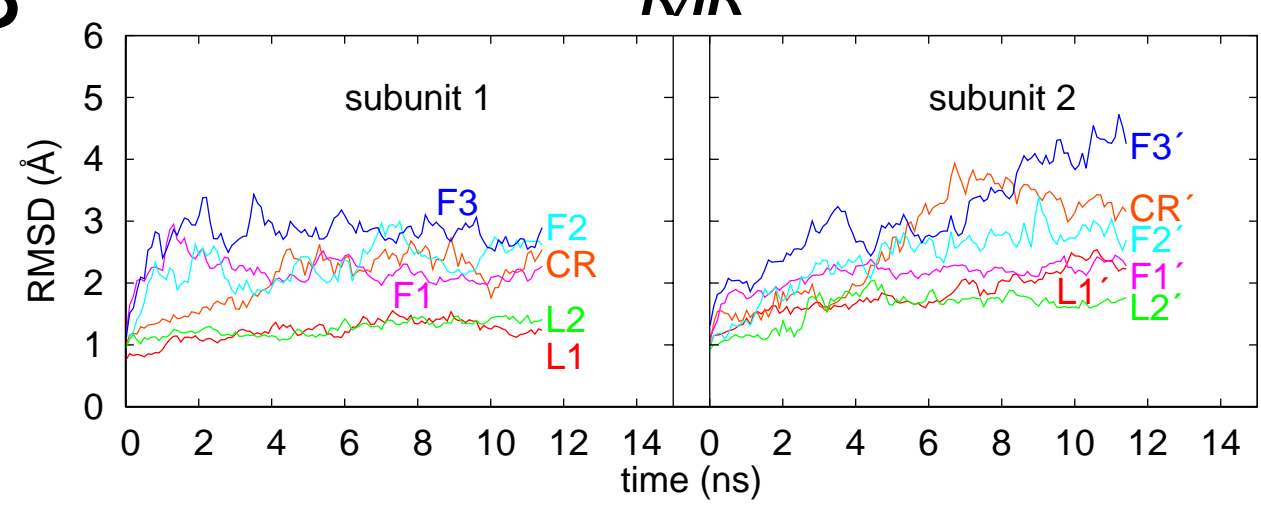

FIGURE S6: RMSD of individual domains of each subunit during MD equilibration of MC-docked models of T-insulin/IR $\Delta \beta$ and R-insulin/IR $\Delta \beta$ complexes. 


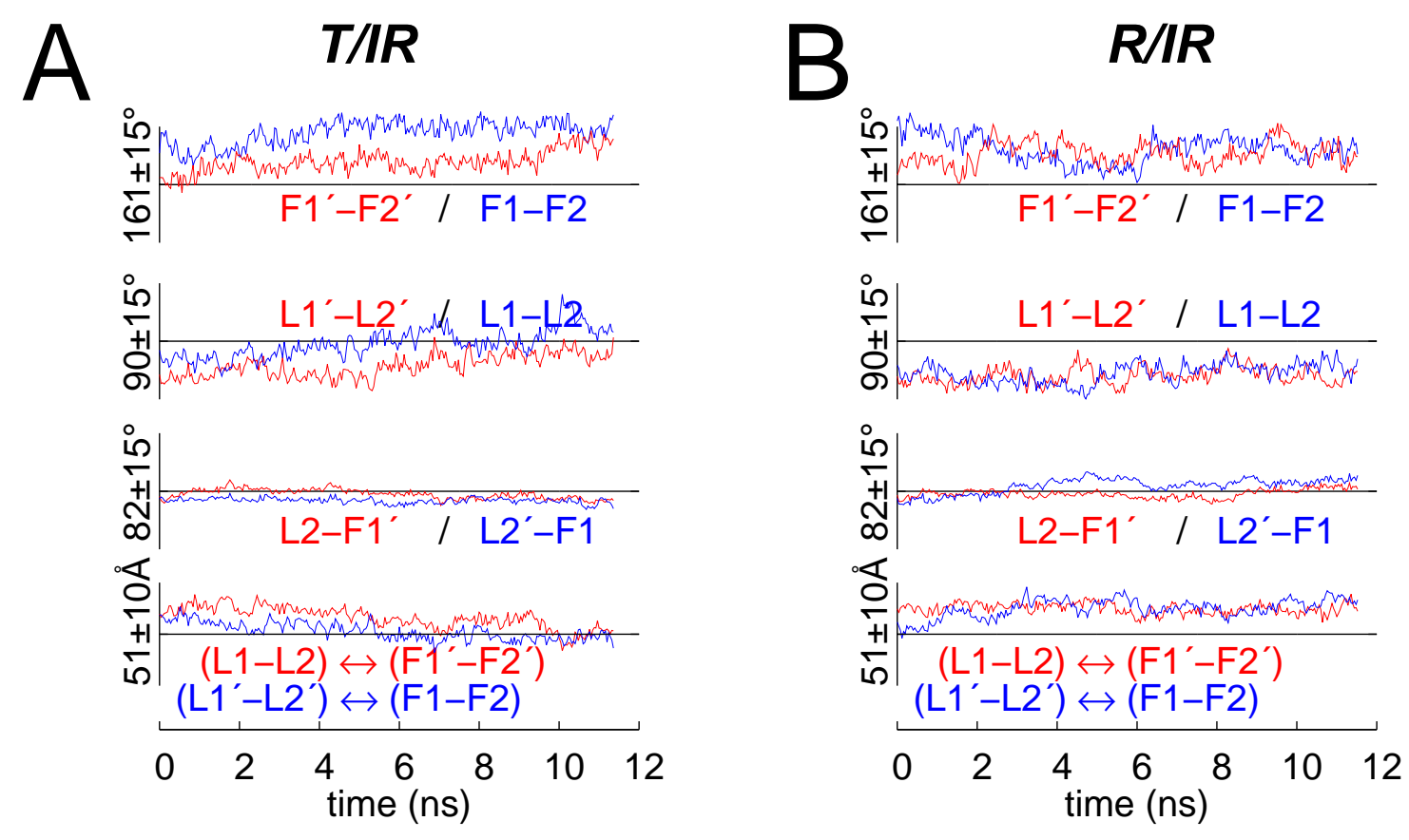

FIGURE S7: Inter-domain hinge-angle and inter-hinge distance conformational metrics during MD equilibration of each insulin/IR $\Delta \beta$ complex. Horizontal lines denote the values measured in the crystal structure (PDB code 3LOH) and vertical lines the scale of each curve.
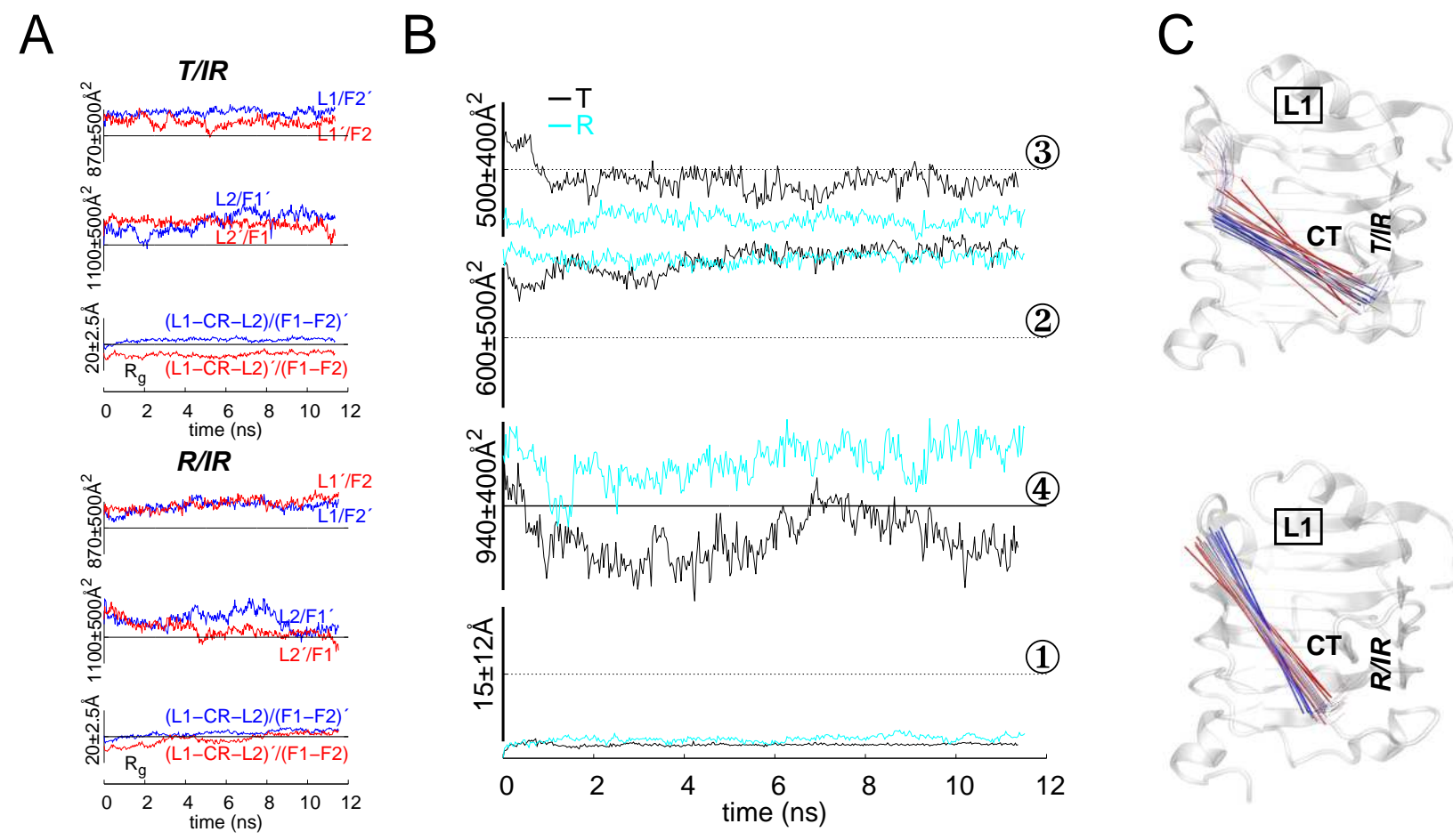

FIGURE S8: ( $A$ ) For each insulin/IR $\Delta \beta$ complex, buried surface area traces between L1/F2 and L2/F1 domains, and the radius of gyration $\left(\mathrm{R}_{\mathrm{g}}\right)$ traces for each binding pocket during MD equilibration. $(B) \mathrm{cf}$. Fig. $2 A$ in the main article for caption. (C) Shown are the snapshots (every $0.5 \mathrm{~ns}$ of the trajectory) of the CT-peptide (labeled CT and shown as a cylindrical tube) highlighted on the surface of the L1 domain (trajectories were aligned based upon L1 domain residues 1-157; $\mathrm{C}_{\alpha}$ ). Some of the terminal residues of CT spontaneously fold/unfold during simulations. Red indicates positions of $\mathrm{CT}$ at the beginning and blue at the end of each trajectory. 


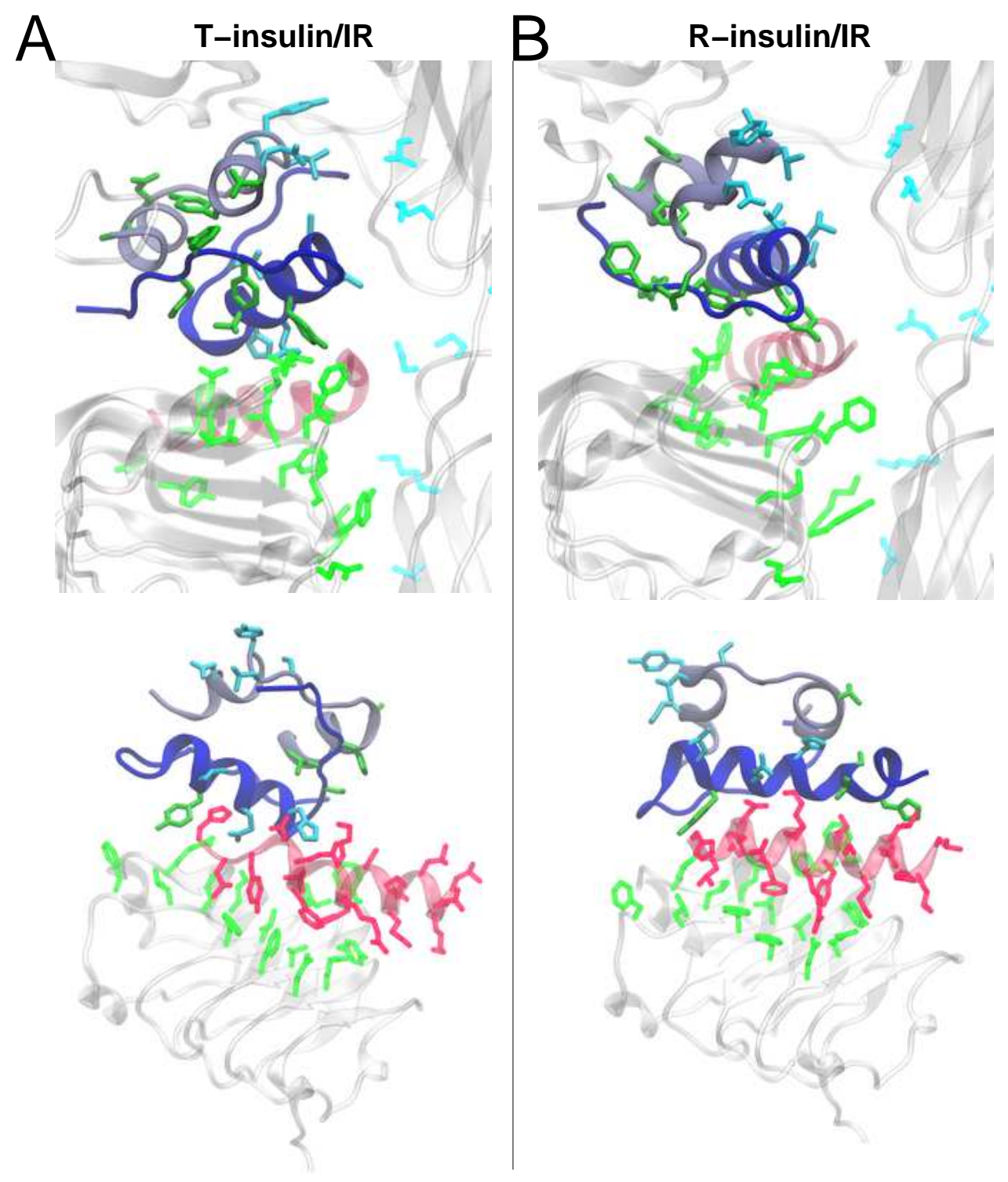

FIGURE S9: Snapshots of contacts between residues of each insulin and IR $\Delta \beta$ after MD equilibration. cf. Fig. 1 in the main article for caption. 
A

$T / / R$

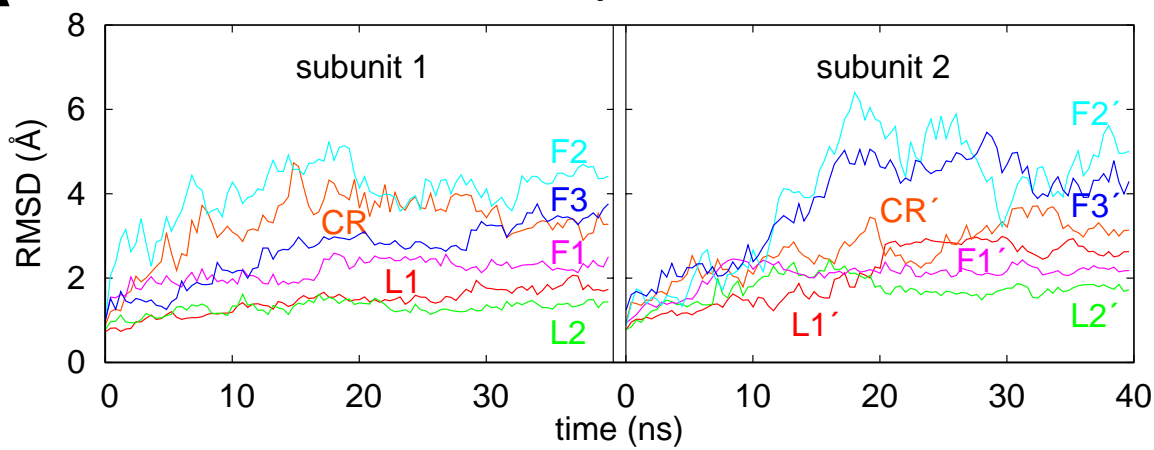

B

$\boldsymbol{R} / \mathbf{R}$

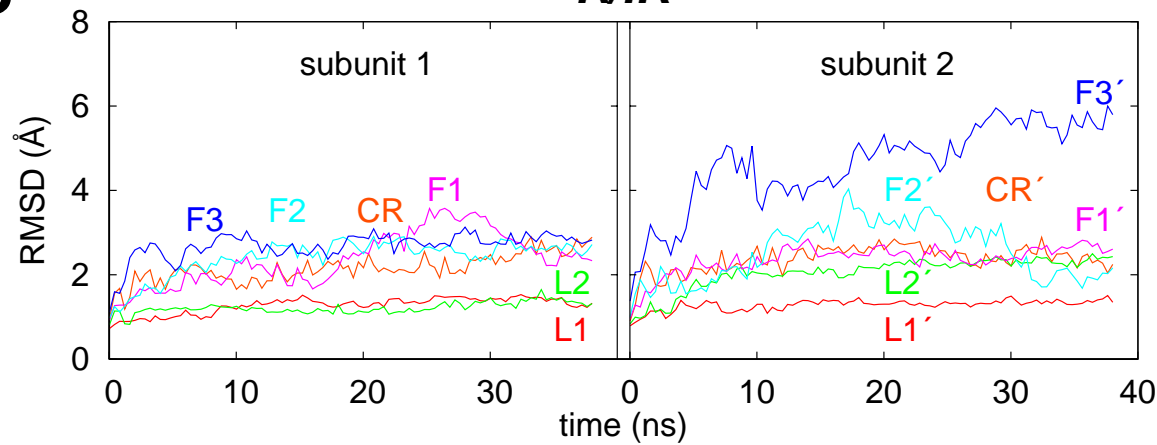

FIGURE S10: RMSD of individual domains of each subunit during a $~ 40-n s$ TAMD simulation (see main manuscript) of each insulin/IR $\Delta \beta$ complex.

A
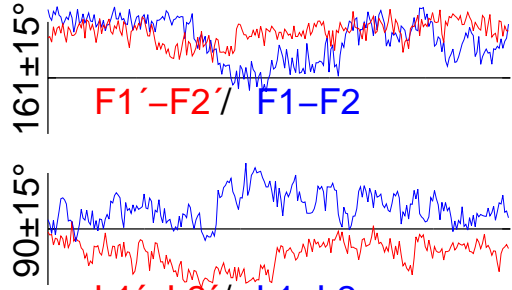

$\mathrm{L} 1^{\prime}-\mathrm{L} 2{ }^{\prime} \mathrm{L} 1-\mathrm{L} 2$

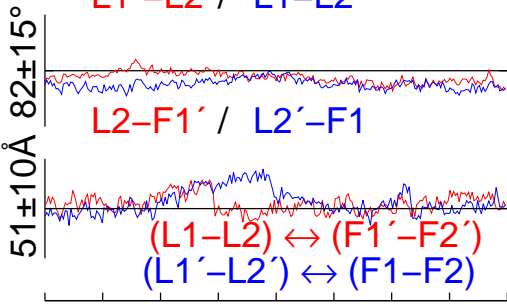

$0 \quad 5 \quad 10152025303540$ time (ns)
B

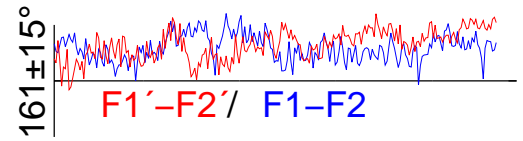

官)

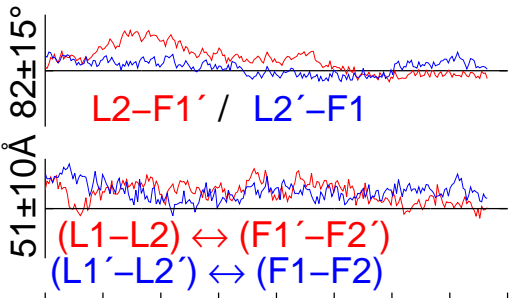

$0 \quad 5 \quad 10152025303540$

FIGURE S11: Inter-domain hinge-angle and inter-hinge distance conformational metrics during a 40-ns TAMD simulation (see main manuscript) of each insulin/IR $\Delta \beta$ complex. Horizontal lines denote the values measured in the crystal structure (PDB code 3LOH) and vertical lines the scale of each curve. 


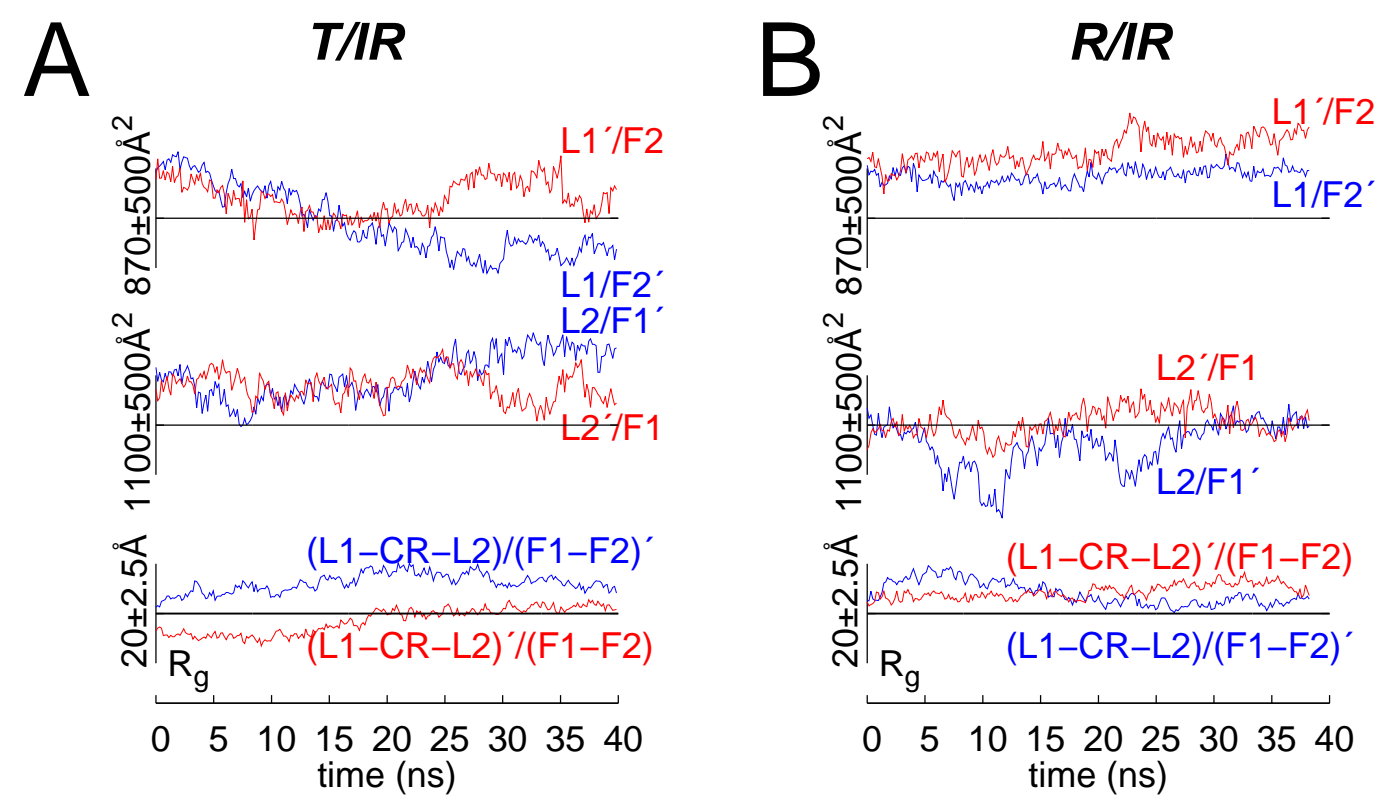

FIGURE S12: For each insulin/IR $\Delta \beta$ complex, buried surface area traces between L1/F2 and L2/F1 domains, and the radius of gyration $\left(\mathrm{R}_{\mathrm{g}}\right)$ traces for each binding pocket during $\sim 40$-ns TAMD simulations.

A
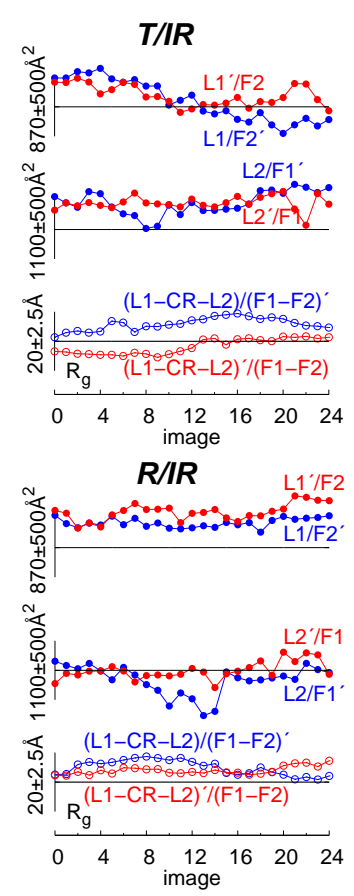

B
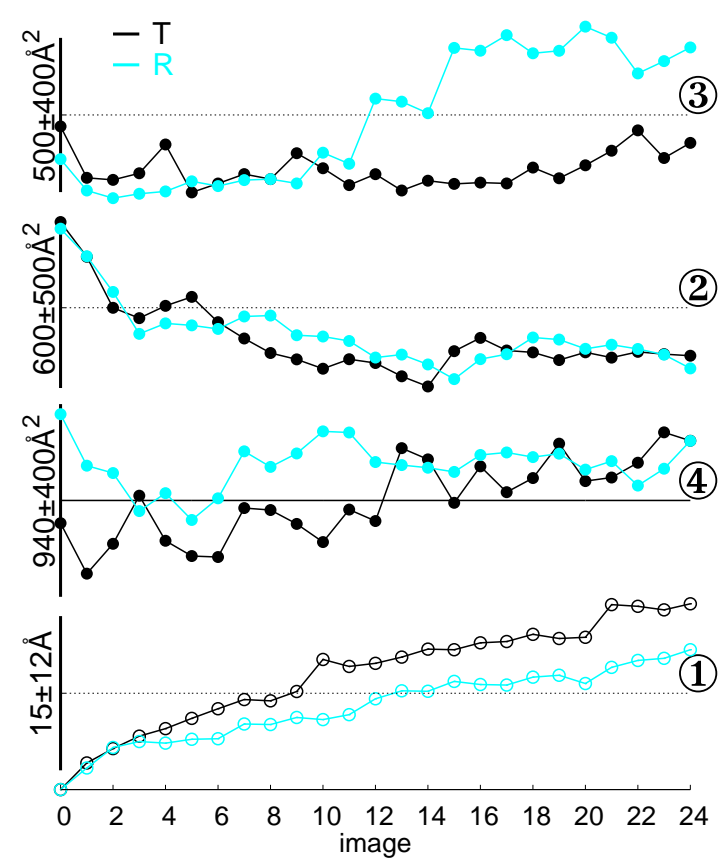

C
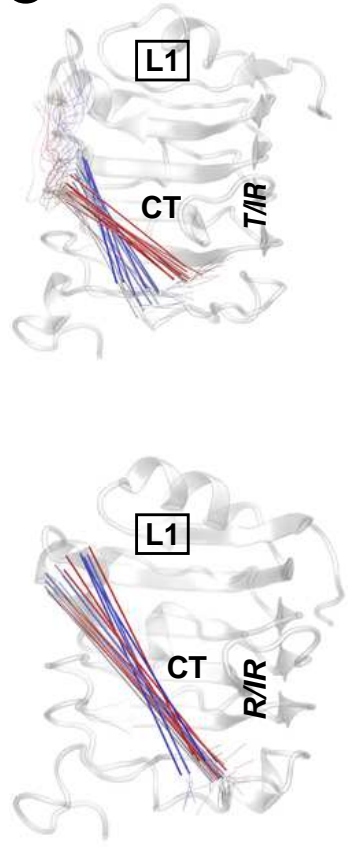

FIGURE S13: Dynamics of receptor, bound-insulin molecules, and the CT-peptide for each minimum-free energy path (MFEP) image. cf. Fig. S8 for caption details. 
A

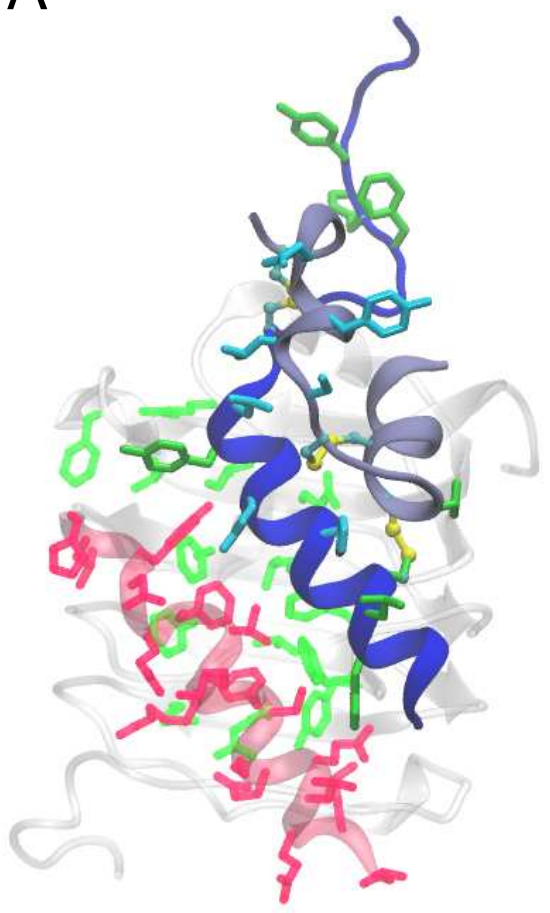

B

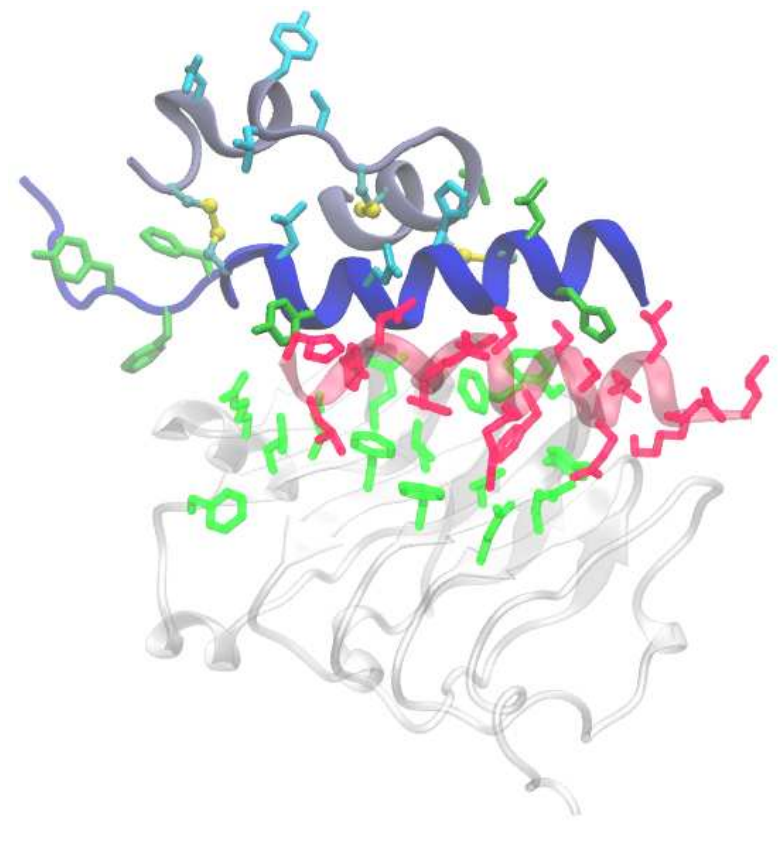

FIGURE S14: Residue-residue contacts between insulin, and L1/CT (IR $\Delta \beta$ ) in the MFEP image\#18 (front and side views on the L1 surface are shown). cf. Fig. 1 in the main article for caption.

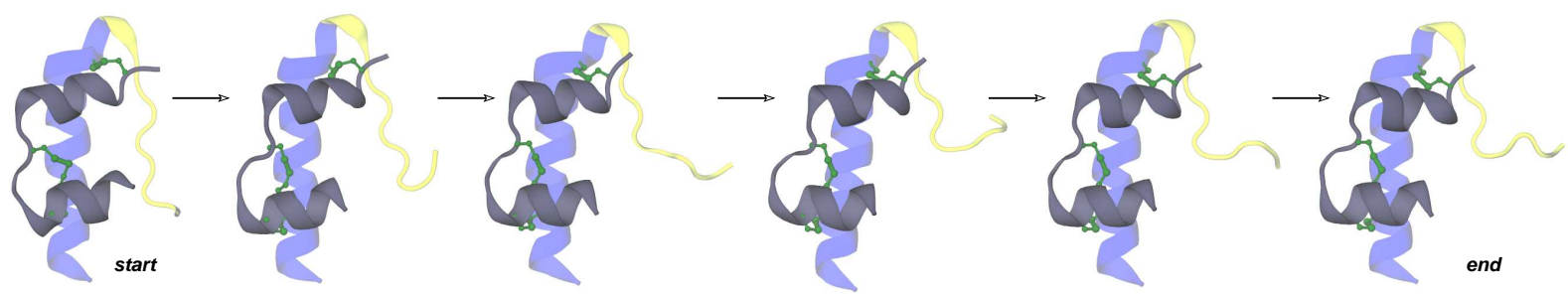

FIGURE S15: Conformational change observed in the C-terminus of the B-chain of R-insulin during a 10-ns unbiased MD simulation is shown. The C-terminus of the B-chain is shown as yellow cartoon with A-chain and B-chain as dark blue and light blue cartoons, respectively. 


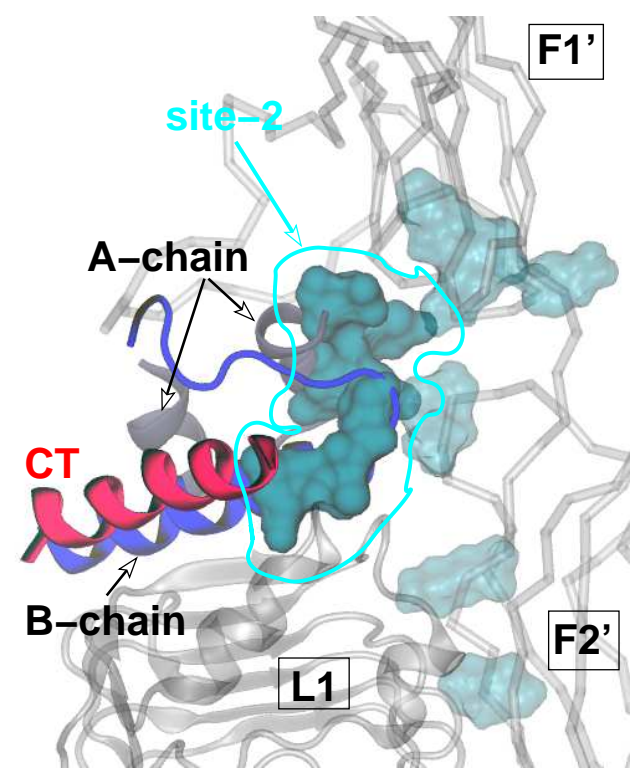

FIGURE S16: Site-2 binding surface of docked R-insulin is highlighted in the displaced-CT model (cf. Fig. 5 in the main manuscript). Receptor domains are labeled inside square boxes with labeled insulin chains, and the CT peptide. A rough boundary showing site-2 receptor binding patch of insulin is highlighted in cyan, while site-2 resodies of IR in (F1-F2)' domains are shown in transparent cyan surfaces.

A

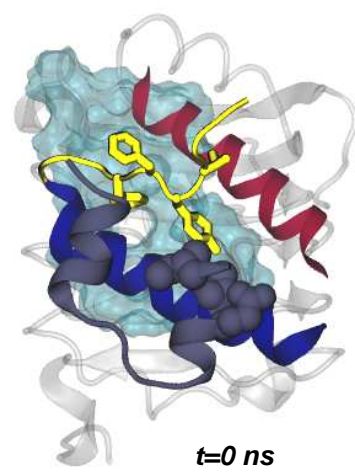



$t=6.6 \mathrm{~ns}$

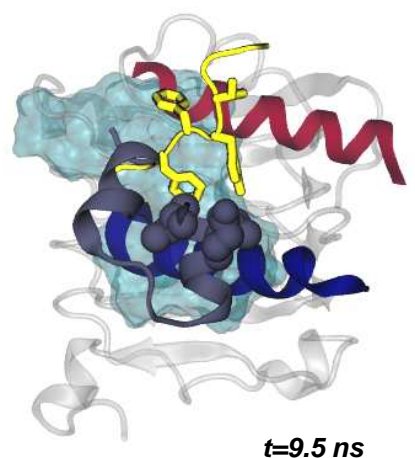

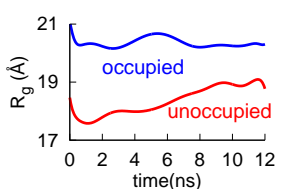



FIGURE S17: $(A)$ Snapshots of docked insulin at three different timepoints from a 12-ns MD equilibration. cf. Fig. 5 in the main manuscript for coloring and labeling details. (B) Traces for buried surface area between docked insulin and L1, radius of gyration $\left(\mathrm{R}_{\mathrm{g}}\right)$ of ligand-occupied and unoccupied pockets of receptor, and distance between the centers-of-mass of fibronectin-III domains (legs) as a function of simulation time (ns) are shown. 


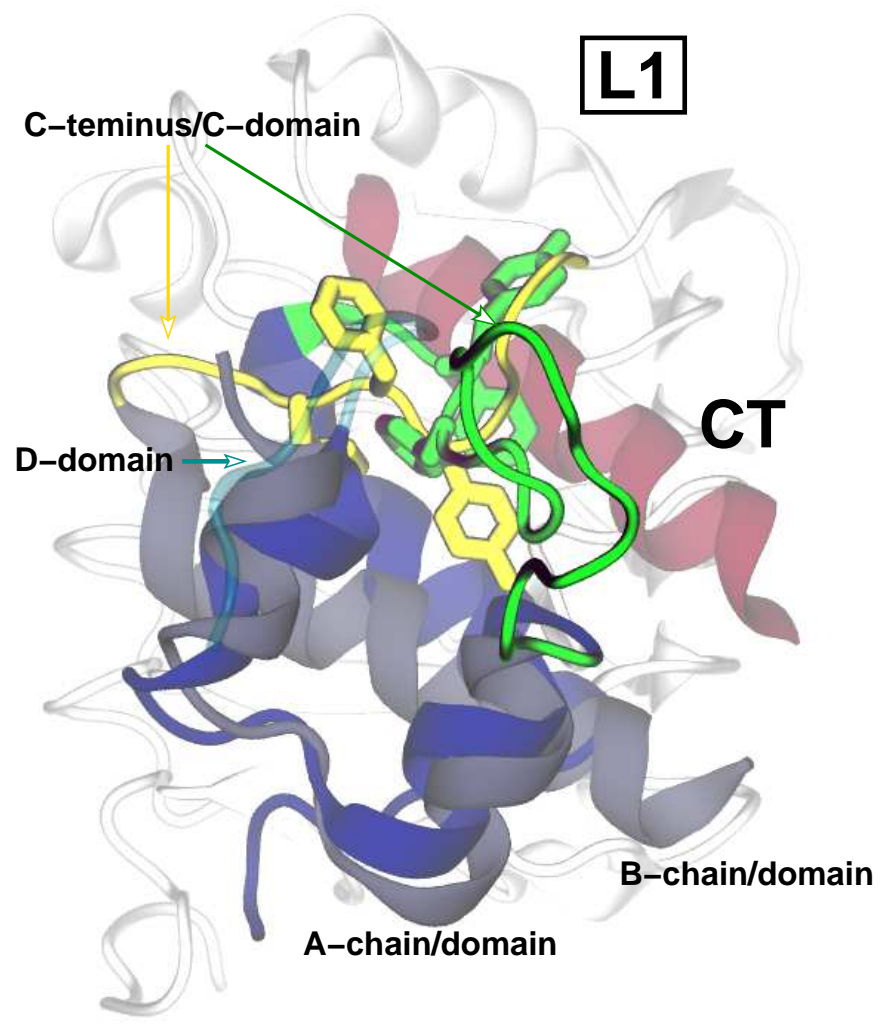

FIGURE S18: Overlay of insulin-like growth factor-1 (IGF1; dark blue cartoon) along with R-insulin (light blue cartoon) on the surface of the L1 domain of IR. IGF1 was aligned on the bound R-insulin monomer in the displaced-CT model (Fig. 5) based upon the A-chain/domain residues. Individual chains/domains of insulin/IGF1 are colored and labeled. The flexible C-terminus of the B-chain of R-insulin is yellow cartoon, and the corresponding C-domain of IGF1 is green cartoon. The sidechains of residues in the FFY /FYF sequence of insulin/IGF1 are also highlighted in respective colored sticks. 
A
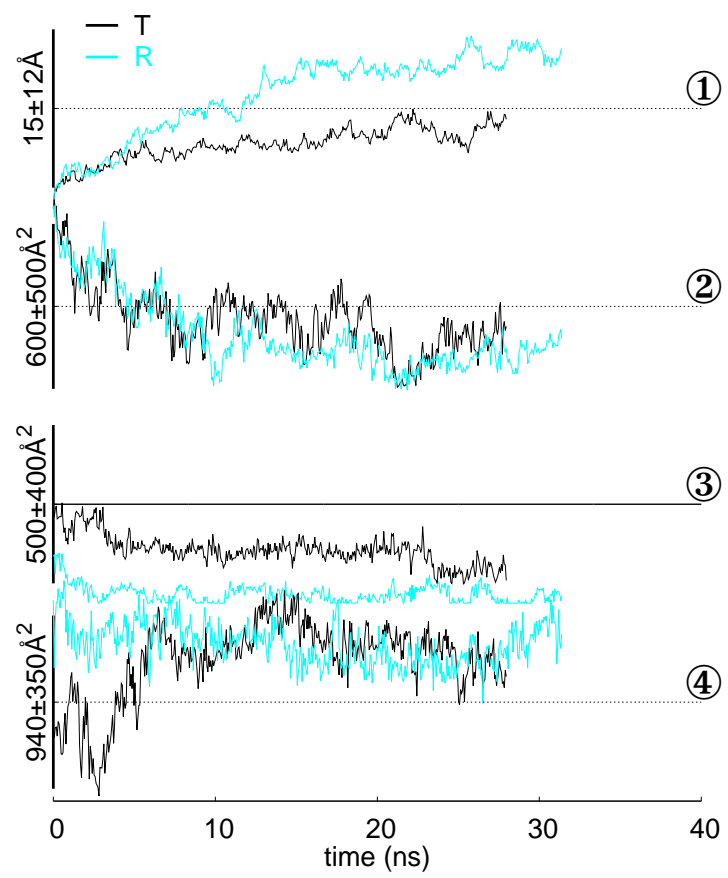

B
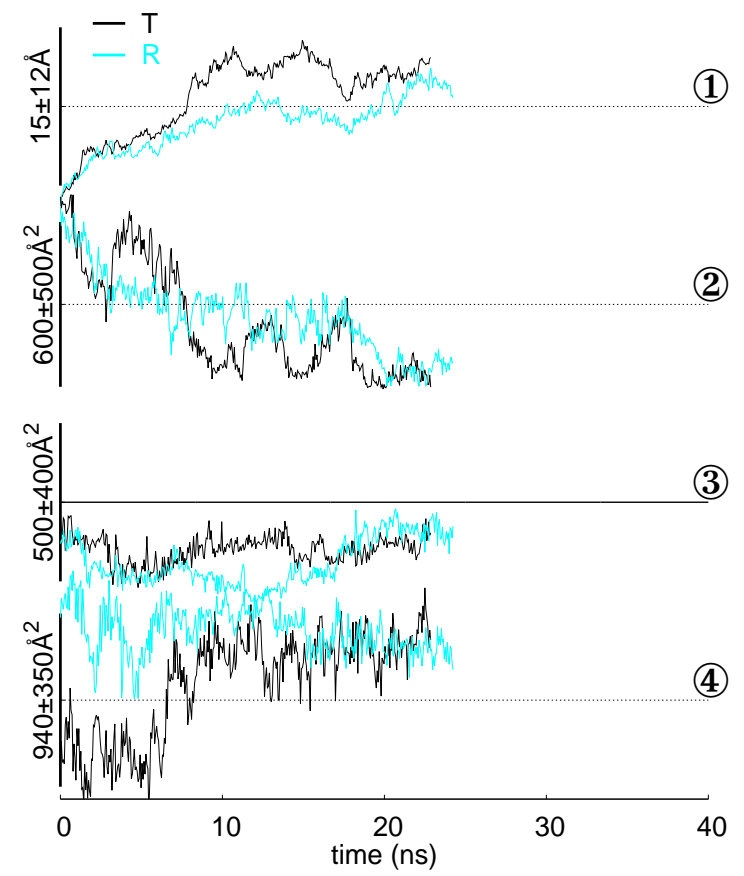

FIGURE S19: RMSD and BSA traces from additional TAMD trajectories for conformational change in the C-terminus of the B-chain of T- and R-insulin: $(A)$ second set of additional TAMD runs (at a fictitious thermal energy $\bar{\beta}^{-1}=6 \mathrm{kcal} / \mathrm{mol}$ ). (B) third set of additional TAMD runs (at a fictitious thermal energy $\bar{\beta}^{-1}=5 \mathrm{kcal} / \mathrm{mol}$ ). cf. Fig. $2 A$ in the main article for caption. 


\section{T-insulin}

\section{R-insulin}


$4.74 A$

FIGURE S20: Comparison of TAMD-generated conformations of each insulin with a novel-insulin analogue. Top left and right panels, respectively for T- and R-insulin, show an overlay of the crystallized conformation of a novel-insulin analogue ([N-Methylated-Ala $\left.{ }^{\mathrm{B} 26}\right]$-insulin; PDB code $2 \mathrm{WRX}$ ) and the TAMD-generated lowest RMSD (with respect to $2 \mathrm{WRX}$ for tail residues $\mathrm{B} 22-\mathrm{B} 30 ; \mathrm{C}_{\alpha}$ ) conformations for each insulin. Bottom left and right panels, respectively for each insulin, show an overlay of tamd-generated conformation of each insulin shown in top panels, and the lowest RMSD (with respect to TAMD-generated conformations of each insulin (top panels ) for tail residues B22-B30; $\mathrm{C}_{\alpha}$ ) conformation of novel-insulin analogue sampled fron an explicit-solvent $10 \mathrm{~ns}$ MD simulation. The insulin molecules are aligned based upon the residues of A- and B-chain (A1-A21 and B8-B19; $\mathrm{C}_{\alpha}$ ). The RMSD ( $\AA$ ) of tail residues are shown on the lower right corner of each panel, and the residues $\mathrm{F}^{\mathrm{B} 24}, \mathrm{~F}^{\mathrm{B} 25}$, and $\mathrm{Y}^{\mathrm{B} 26} / \mathrm{A}^{\mathrm{B} 26}$ are shown in sticks. 